\title{
Reticular chemistry in electrochemical carbon dioxide reduction
}

\author{
Yanfang Wang ${ }_{1^{*}}^{1,2,4}$, Yuexiang $\mathrm{Li}^{3 \dagger}$, Zhenyu Wang ${ }^{1}$, Phoebe Allan ${ }^{4^{*}}$, Fucai Zhang ${ }^{2^{*}}$ and \\ Zhouguang $\mathrm{Lu}^{1^{*}}$
}

\begin{abstract}
Electrochemical $\mathrm{CO}_{2}$ reduction (ECR) represents a promising strategy for utilizing $\mathrm{CO}_{2}$, an industrial waste, as an abundant and cheap carbon source for organic synthesis as well as storing intermittent renewable electricity from renewable sources. Efficient electrocatalysts allowing $\mathrm{CO}_{2}$ to be reduced selectively and actively are crucial since the ECR is a complex and sluggish process producing a variety of products. Metal-organic frameworks (MOFs) and covalentorganic frameworks (COFs) have emerged as versatile materials applicable in many fields due to their unique properties including high surface areas and tunable pore channels. Besides, the emerging reticular chemistry makes tuning their features on the atomic/molecular levels possible, thereby lending credence to the prospect of their utilizations. Herein, an overview of recent progress in employing framework material-based catalysts, including MOFs, COFs and their derivatives, for ECR is provided. The pertinent challenges, future trends, and opportunities associated with those systems are also discussed.
\end{abstract}

Keywords: reticular chemistry, MOFs, COFs, electrochemistry, carbon dioxide reduction

\section{INTRODUCTION}

Ever since the Industrial Revolution, traditional fossil fuels have become the primary source for global energy supply underpinning the global economy. There is no doubt that energy supply is a key issue for the sustainable development of modern society. Especially when encountering with the contradiction between the growing demand for energy and the depletion of fossil fuels, it is paramount to develop new and sustainable sources of energy [1]. Worse, the continuing reliance on fossil fuels by developed countries and the increasing demand for energy by emerging countries make the emission of carbon dioxide into the atmosphere a serious global problem [2]. Energy security and environmental concerns have provided impetus for finding alternative energy sources. During the past decades, tremendous research efforts, both in academic and industrial communities, have been devoted to harvesting energy from the sun, wind, tide and other renewable sources [3-5]. Although these are active areas of research and development, and are being used in some countries, they still constitute a minority of the global energy supply because of various issues related to cost, storage and scalability [6]. It is therefore reasonable to anticipate that fossil fuels will continue to be important to, if not dominate, the world's energy landscape for some time to come. Therefore, it is urgent in the short term to address carbon dioxide emissions, while continuing to develop alternative fuels as a long-term solution.

From the perspective of substantially mitigating anthropogenic $\mathrm{CO}_{2}$ emissions, carbon capture and storage (CCS) and carbon capture and utilization (CCU) have been regarded as feasible strategies [7-9]. Typically, CCS includes various technologies which capture $\mathrm{CO}_{2}$ at some stage from processes in power generation, cement manufacturing, iron/steel making and natural gas treatment. Then, the captured $\mathrm{CO}_{2}$ is pressurized, transported and injected into stable geological sites, being trapped for thousands of years [10]. For smaller/medium emitters where geological sequestration is not viable, mineral

\footnotetext{
${ }^{1}$ Department of Materials Science and Engineering, Southern University of Science and Technology, Shenzhen 518055, China

${ }^{2}$ Department of Electronic and Electrical Engineering, Southern University of Science and Technology, Shenzhen 518055, China

${ }^{3}$ Alibaba Cloud, Alibaba (Shenzhen) Technology Co., Ltd, Shenzhen 518000, China

${ }^{4}$ School of Chemistry, University of Birmingham, Birmingham B15 2NT, UK

${ }^{\dagger}$ Wang Y and Li Y contributed equally to this work.

* Corresponding authors (email: P.Allan@bham.ac.uk (Allan P); zhangfc@sustech.edu.cn (Zhang F); luzg@sustech.edu.cn (Lu Z))
} 
carbonation (MC) is emerging as a promising CCS technology, in which $\mathrm{CO}_{2}$ reacts with calcium- and/or magnesium-containing materials to form stable carbonates [11]. Although most of the components of the CCS chain have been demonstrated to be individually viable, their integration is challenging and continues to be an uphill battle. On the other hand, as a relatively benign material, $\mathrm{CO}_{2}$ could be utilized in many fields and converted into a wide variety of end products. Thus, CCU represents an appealing opportunity and opens a new perspective to the conversion of waste product into highvalue end products, moving the $\mathrm{C}$-economy from a linear trend to a carbon-neutral full cycle $[12,13]$.

Nowadays, with increasing electricity generated from renewable energy sources, the electrochemical $\mathrm{CO}_{2}$ reduction (ECR) has attracted much attention and particular interest as it can simultaneously contribute to the utilization of $\mathrm{CO}_{2}$ and to the storage of electric energy [14-16]. The ECR has several advantages including controllable processes by adjusting potentials, minimized chemical consumption and feasibility to scale up. However, due to the large reorganization energy associated with the transformation from the stable linear $\mathrm{CO}_{2}$ molecule to the bent $\mathrm{CO}_{2}{ }^{-}$radical anion, this reduction process only occurs at a potential as negative as $-1.90 \mathrm{~V}$ vs. reversible hydrogen electrode (RHE) [17]. Also, the direct electrochemical reduction of $\mathrm{CO}_{2}$ results in a variety of products, which mainly depend on catalysts and the reaction media. Generally, ECR may undergo, for example, two-, six-, and eight-electron reduction pathways in aqueous and non-aqueous phases with different standard electrode potentials, forming a wide variety of compounds including $\mathrm{CO}, \mathrm{HCOOH}$ and hydrocarbons/ alcohols [18-20]. Besides, the ECR in aqueous solutions faces challenges from the competing hydrogen evolution reaction (HER). Therefore, exploring appropriate electrocatalysts is of critical importance to obtain high energy efficiency and selective production in ECR. The majority of existing electrocatalysts for ECR can be divided into two groups: heterogeneous and homogeneous (more details will be introduced in the second part). Typically, the performances of an electrocatalyst could be evaluated in terms of selectivity and activity $[21,22]$, which determine the energy efficiency of a process. The selectivity dictates the product and purity thereof and is directly linked to the chemical nature and geometric property of the catalyst in relation to the binding affinities to $\mathrm{CO}_{2}$ and reaction intermediates. The activity providing the turnover is intrinsically determined by the relative binding affinities to $\mathrm{CO}_{2}$ and the product in a manner that is de- scribed by the volcano plot. The number of the accessible active sites, which correlate to the surface area and metrics of the catalyst structures, also determines the activity in a practical sense. Currently, the grand challenge in ECR lies in the fact that a single catalytic system must control the interplay between selectivity and activity. Furthermore, it also needs to possess a long-term stability for commercial applications.

In the past decades, with the development of reticular chemistry, metal-organic frameworks (MOFs) and covalent-organic frameworks (COFs) have emerged as attractive materials being capable of addressing various issues [23-25]. By virtue of their unique properties in terms of controllable porosity and ultra-high specific surface areas, they exhibit versatile applications in many fields including gas storage and separation, sensors, biomimicry, energy conversion and storage, drug delivery, and catalysis [26-35]. Further, their structures and properties can be controlled precisely by varying the constituent geometry as well as by grafting functional groups, exemplifying the manner in which reticular chemistry is practiced [36-38]. Recently, interconnecting metal complexes with organic ligands to form stable organic frameworks gives a novel way of immobilizing homogenous catalysts. Combining the merits of heterogeneous and homogeneous catalysts, such materials possessing high surface areas and porous structures are capable of performing effectively for ECR [39]. In particular, the uniform dispersion of active sites in those frameworks avoids aggregation and hence maximizes their utilization, while the interconnected frameworks make each single site accessible to substrate molecules. Moreover, the microenvironment around the active sites and porous structures can be tuned to favor a specific reaction pathway, which in turn benefits the activity and selectivity of ECR.

Considering those appealing characteristics of MOFs and COFs, a running theme of this review pertains to how the reticular chemistry can be applied for designing effective electrocatalysts. This review begins with a short introduction of possible pathways of ECR and traditional electrocatalysts for ECR. Then, we will present recent progresses in immobilizing homogeneous catalysts by constructing MOFs or COFs and show how those framework materials can be precisely modified on an atomic or a molecular level to improve their activity and selectivity towards ECR. Also, some other framework materials and their derivatives reported for ECR will be included. Finally, future opportunities and challenges of employing MOFs-based materials as ECR catalysts will be 
discussed.

\section{THE PATHWAYS OF ECR}

ECR is a reaction with multiple steps and might undergo complicated pathways towards different products. In aqueous solutions, the reduction of $\mathrm{CO}_{2}$ is always accompanied by the oxygen evolution reaction (OER) on the anode and needs to compete with the parasitic HER on the cathode (Fig. 1).

Although various physical and chemical processes might be involved, there are generally three steps in the ECR: adsorption, activation and desorption. In an ECR system, to reach the electrode surface on which the electrocatalysts are loaded and electrochemical reactions occur, the gaseous $\mathrm{CO}_{2}$ firstly dissolves in solutions, reacts with solvents and forms $\mathrm{CO}_{2}$-saturated electrolytes. The initial binding model between solvated $\mathrm{CO}_{2}$ and the electrocatalyst, which might be affected by factors such as local concentration of $\mathrm{H}^{+}(\mathrm{pH})$ and overpotential, determines the primary products in the early stages of ECR [40]. For instance, in acidic solutions, solvated $\mathrm{H}^{+}$is more likely to accumulate on the surface of electrode and bind to the electrocatalyst in the form of surface hydrogen $(* \mathrm{H}$, *refers to the atom bound to the catalyst), which then reacts with physisorbed $\mathrm{CO}_{2}$ along with one electron transfer to form formate through an intermediate $* \mathrm{HCOO}$ or $* \mathrm{OCHO}$ (Fig. 2) [41,42]. It is noteworthy to indicate that HER usually occurs along with formate production because both reactions need $* \mathrm{H}$. However, in neutral electrolytes, the solvated $\mathrm{CO}_{2}$ is directly physi-

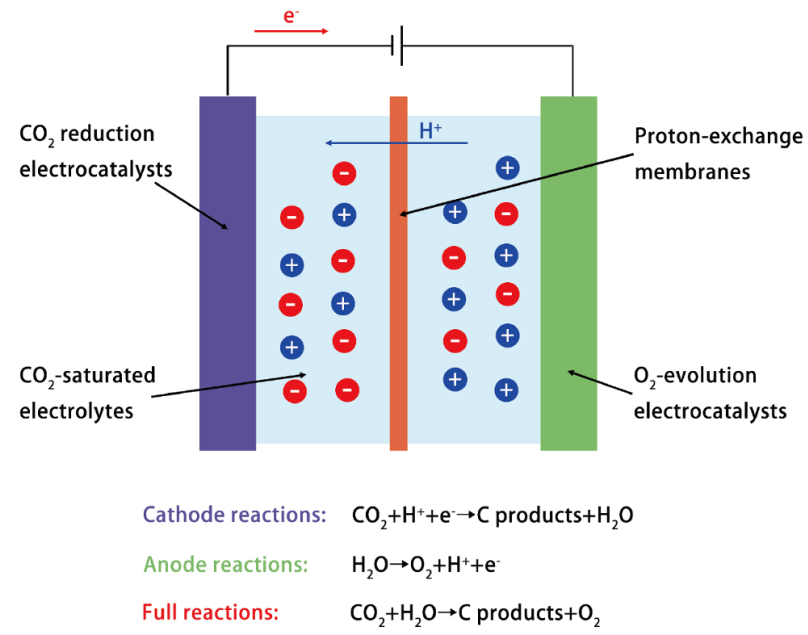

Figure 1 A typical system for ECR. ECR and OER occur on the cathode and anode, producing $\mathrm{C}$ products and oxygen, respectively. Protonexchange membranes allow proton transfer across the electrolyte and prevent unwanted ion migrations. sorbed to the electrode before its further reduction to chemisorbed $\mathrm{CO}_{2}\left({ }^{*} \mathrm{CO}_{2}{ }^{\delta-}\right)$, ${ }^{*} \mathrm{COOH}$ and $* \mathrm{CO}$, in which *CO is believed to be the key intermediate for the formation of CO and high value-added hydrocarbons [43]. While many other factors are important, the binding energy between the catalysts and * $\mathrm{CO}$ to some extent determines whether it will be released in $\mathrm{CO}$ or participates in further reductions. Generally, catalysts showing weak bonds to ${ }^{*} \mathrm{CO}$ are more likely to produce $\mathrm{CO}$ in ECR because gaseous $\mathrm{CO}$ can desorb from the binding sites more easily. With an appropriate catalyst for its further reduction, the bound ${ }^{*} \mathrm{CO}$ might undergo two types of reactions: hydrogeneration and $\mathrm{C}-\mathrm{C}$ coupling, resulting in $\mathrm{C}_{1}$ and $\mathrm{C}_{2} / \mathrm{C}_{3}$ products, respectively. The first hydrogeneration might occur on $\mathrm{C}$ or $\mathrm{O}$ in ${ }^{*} \mathrm{CO}$, producing either ${ }^{*} \mathrm{CHO}$ or ${ }^{*} \mathrm{COH}$, and theoretical calculation suggests the later intermediate on the basis of activation energy $[44,45]$. Further hydrogeneration and dehydration lead to the formation of several intermediates (e.g., ${ }^{*} \mathrm{CHOH},{ }^{*} \mathrm{CH}_{x},{ }^{*} \mathrm{OCH}_{x}$ ) and final products $\left(\mathrm{CH}_{4}\right.$ and $\left.\mathrm{CH}_{3} \mathrm{OH}\right)$ [46].

For multicarbon products, ${ }^{*} \mathrm{OC}-\mathrm{CO}$ is believed to be the key intermediate generated via the dimerization of *CO. Through several proton-coupled electron transfers (PCETs), in which the proton and electron simultaneously transfer to the adsorbed species, *OC-CO will be reduced to $\mathrm{C}_{2} \mathrm{H}_{4}, \mathrm{C}_{2} \mathrm{H}_{5} \mathrm{OH}$ and other $\mathrm{C}_{2}$ products $[47,48]$. In fact, the formation of $\mathrm{C}-\mathrm{C}$ bonds varies in different reaction conditions. Recent experimental results and theoretical calculation indicate that $\mathrm{C}-\mathrm{C}$ coupling prefers to occur at relatively low overpotential and in alkaline electrolytes because HER and hydrogeneration will dominate at more negative potentials or acidic $\mathrm{pH}$ [49]. The ${ }^{*} \mathrm{CO}$ coverage has also been proved to be pivotal in tuning the ECR pathways. Generally, increasing the *CO coverage promotes $\mathrm{C}-\mathrm{C}$ coupling because concentrated *CO intermediates repulse each other and decrease their binding energy to catalysts [50,51]. In other words, both the cleavage of catalyst- $\mathrm{C}$ bonds and the formation of $\mathrm{C}-$ $\mathrm{C}$ bonds are favored when the intermediates are bound neither too strongly nor too weakly to the catalysts. Besides, with high ${ }^{*} \mathrm{CO}$ coverage, ${ }^{*} \mathrm{H}$ adsorption will be constrained, suppressing competitive HER and hydrogeneration.

Admittedly, considering the complexity of the reaction mechanism and the numerous possibilities at every branching point in $\mathrm{C}-\mathrm{C}$ coupling processes, achieving a high selectivity towards one specific multicarbon product is challenging. However, through precise design, many recent attempts have been proved to be successful in al- 


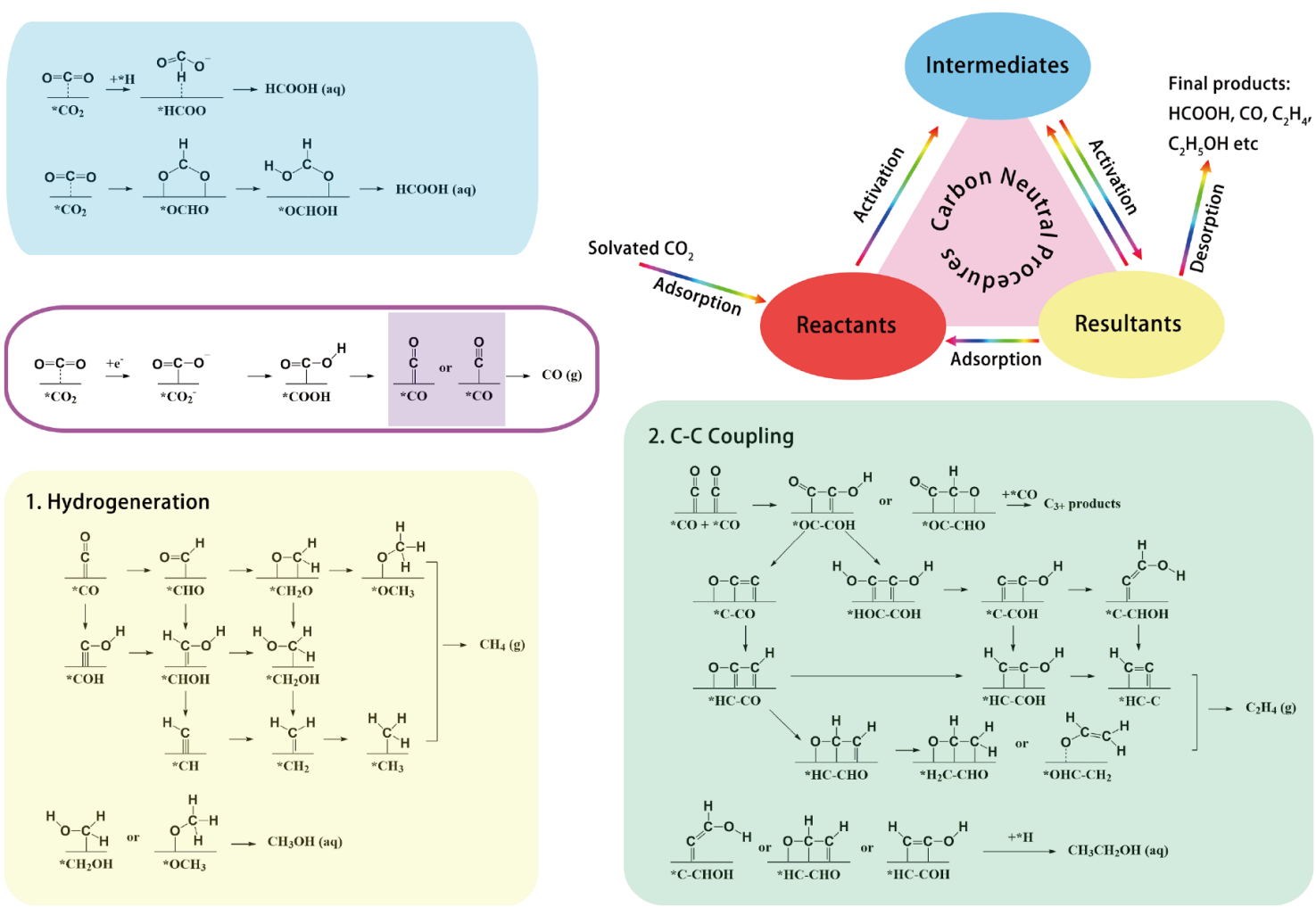

Figure 2 Possible pathways from gaseous $\mathrm{CO}_{2}$ to valuable $\mathrm{C}$ products. Every step refers to a PCET, in which the proton and electron simultaneously transfer to the adsorbed species.

tering the ECR selectivity [52-55]. For example, a record high Faradaic efficiency (FE) of $72 \%$ from $\mathrm{CO}_{2}$ to $\mathrm{C}_{2} \mathrm{H}_{4}$ was achieved by producing *CO-rich local environment through molecular tuning [56]. Thus, by further understanding the mechanism of ECR and by altering the reaction environment through precise design of electrocatalysts, electrolytes and their interfaces, it is possible to shut down unwanted pathways and increase the catalytic activity towards desired products.

\section{TRADITIONAL ELECTROCATALYSTS FOR ECR}

In this section, some typical examples regarding employing heterogeneous and homogeneous electrocatalysts for ECR are included, aiming to show the advantages and disadvantages of different systems rather than to depict the whole picture of traditional electrocatalysts.

\section{Heterogeneous systems}

Among heterogeneous systems, in the early stage, polycrystalline monometallic catalysts were widely evaluated because of their simple structures and facile syntheses $[57,58]$. In those systems, both experimental and theo- retical results have demonstrated that the adsorption free energies of $\mathrm{CO}_{2}$, intermediates and products to the underlying structure of the metals have great impact on the product distribution [59-63]. Commonly, their binding affinities are affected by several features including size, oxidation state, structure and crystal phase. For instance, an oxide-derived Au exhibited highly selective and stable $\mathrm{CO}_{2}$ reduction to $\mathrm{CO}$ in aqueous solutions at lower overpotential than that of its polycrystalline counterpart, which was attributed to the dramatically increased stabilization of the $\mathrm{CO}_{2} \cdot{ }^{-}$intermediate on such electrodes [64]. Also, recent progresses have demonstrated that partially reduced $\mathrm{SnO}_{x}$ and $\mathrm{CoO}_{x}$ are able to catalyze the ECR to $\mathrm{CO}$ with high selectivity while Co and $\mathrm{Sn}$ are inclined to catalyze the HER [65-67]. However, the preferred path way for ECR is not determined solely by energetics, and the reaction kinetics also play pivotal roles in determining the selectivity [68-71]. For example, a nanoporous $\mathrm{Ag}$ electrocatalyst showed approximately $92 \%$ selectivity towards $\mathrm{CO}$ at a rate over 3000 times higher than that of its polycrystalline counterpart in an aqueous bicarbonate medium due to its large electrochemical surface area and the greater stabilization of 
$\mathrm{CO}_{2} \cdot{ }^{-}$intermediate on the highly curved surface [72]. A dramatic increase in $\mathrm{pH}$ within this porous electrode could also contribute to this enhanced ECR selectivity since the ECR is less sensitive to $\mathrm{pH}$ than the competing HER. From these perspectives, fabricating proper structures facilitating charge and mass transports for a desired reaction pathway is also of great importance to obtain high-performance electrocatalysts.

To maximize the performance in virtue of advantages from different metals and their synergistic effects, bimetallic electrocatalysts have drawn tremendous research attentions [73-75]. For example, the ordered gold-copper monolayers showed superior catalytic performance for ECR, benefiting from their combined electric and geometric effects [76]. Besides, transition metal dichalcogenides $\left(\mathrm{MoS}_{2}, \mathrm{WS}_{2}, \mathrm{MoSe}_{2}, \mathrm{MoTe}_{2}\right.$ and so on) have also been explored [77-79]. The layered $\mathrm{MoS}_{2}$ could reduce $\mathrm{CO}_{2}$ to $\mathrm{CO}$ selectively in an aqueous electrolyte solution containing an ionic liquid (IL), in which the Mo-terminated edges were regarded as active sites by participating in the electron-transfer reactions [80]. Notwithstanding their activity and selectivity towards ECR, metal-based electrocatalysts usually present poor durability for longterm reactions, a drawback that hinders their practical applications.

As low-cost and metal-free materials, carbon materials have been widely studied as electrocatalysts for many important reactions because of their excellent electrical properties, tunable structures and superior stabilities [8185]. However, for ECR, carbon materials usually exhibit negligible activity and catalyze the competing HER in preference. Fortunately, heteroatoms (N, B, P, etc.) doping into the carbon network could induce the rearrangement of charge density and introduce active sites, so that the adsorption of the $* \mathrm{COOH}$ intermediate (an up-hill elementary step in the ECR pathway) could be facilitated [86-88]. For example, N-doping of carbons (graphene, carbon nanotubes and carbon nanofibers, etc.) breaks up the neutrality of carbon materials through introducing extra electrons and hence enhances the activity towards ECR to two-electron products such as $\mathrm{CO}$ in an IL containing aqueous electrolyte solution or formic acid in an aqueous bicarbonate solution) [89]. Higher order hydrocarbons have also been produced with $\mathrm{N}$-doped carbon electrocatalysts. Notably, N-doped graphene quantum dots (NGQDs), being able to suppress the HER, could catalyze $\mathrm{CO}_{2}$ reduction to ethanol and ethylene with a total $\mathrm{FE}$ of $90 \%$ in an aqueous bicarbonate solution due to the ultra-high density of edge sites along with the $\mathrm{N}$ defect [90].
Owing to their large surface areas and excellent network conductivity, carbon materials have also been used as skeleton structures to anchor and stabilize metal-based materials, which in turn could deliver enhanced catalytic activity towards ECR [91-93]. Besides, carbon supported metal hybrid materials have been demonstrated to be more active for $\mathrm{C}-\mathrm{C}$ coupling by creating a high $\mathrm{CO}_{2}$ pressure at the triple-phase boundary, thus generating $\mathrm{C}_{2}$ or $\mathrm{C}_{3}$ products [94]. Recently, incorporating metals in $\mathrm{N}$ doped carbons gives a class of materials termed as M-N$\mathrm{C}$, where $\mathrm{M}$ is transition metal such as $\mathrm{Fe}$ and/or $\mathrm{Mn}$, and they have been shown to be cost efficient alternatives to noble metal catalysts for syngas production [95-98].

In general, with the recent advances in nanoengineering technology, various heterogeneous catalysts with controllable size, composition or morphology can now be designed to control specific reaction pathways in order to achieve high selectivity and activity. However, the mechanistic details in those systems have not been obtained. Consequently, the rate-limiting and key selectivity-determining steps are still controversial.

\section{Homogeneous systems}

Molecular catalysts consisting of a variety of redox-active metal complexes have also been widely investigated for ECR [99-102]. In those homogeneous systems, catalytically active sites and reaction intermediates are comparatively easy to characterize, thereby better elucidating the mechanistic details of the catalytic reaction. Moreover, those catalysts could be modulated at a molecular level to suit for desired catalytic processes [103].

For example, as one class of the most thoroughly investigated molecular catalysts, metalloporphyrin systems usually exhibit high activity and selectivity for reducing $\mathrm{CO}_{2}$ to $\mathrm{CO}$. Specifically, $\mathrm{CO}_{2}$ molecules bind to the nucleophilic $\mathrm{Fe}(0)$ centers of iron (0) tetraphenylporphyrin, electrochemically generated from iron (II) tetraphenylporphyrin (FeTPP). $\mathrm{CO}_{2}$ in the $\mathrm{Fe}(0)-\mathrm{CO}_{2}$ adduct was further protonated with Lewis acids or weak Brönsted acids and reduced to afford $\mathrm{CO}$ upon cleavage of a $\mathrm{C}-\mathrm{O}$ bond [104]. Its catalytic performance was improved by introducing different substituents on the periphery of the porphyrin ring. Introducing phenolic groups in FeTPP could accelerate the protonation process by creating a high local concentration of proton and stabilize the $\mathrm{Fe}(0)$ $\mathrm{CO}_{2}$ adduct through $\mathrm{H}$ bonding [105]. Further substitution of the above material by fluorine $(\mathrm{F})$ was favorable to lowering the reaction overpotential due to the inductive effect of electronegative $\mathrm{F}$ atoms promoting the reduction of the molecule to the $\mathrm{Fe}(0)$ state [106]. Commonly, those 
molecular catalysts were investigated for $\mathrm{CO}_{2}$-to- $\mathrm{CO}$ conversion in aprotic media (mostly $\mathrm{N}, \mathrm{N}$-dimethyllformamide (DMF) and acetonitrile) because they also catalyze the competing HER in aqueous media. Excitingly, substitution of four paraphenyl hydrogens by trimethylammonio groups in FeTPP provided a watersoluble molecule being able to catalyze ECR with a high selectivity of $\mathrm{CO}$ in $\mathrm{pH}$-neutral water, which is promising for practical applications when considering its integration with the water oxidation anode in a full-cell [107].

Although those homogeneous catalysts could deliver good selectivity and activity for ECR, they usually suffer from drawbacks including product isolation, catalyst recovery, and poor stability $[108,109]$. Also, they are only electro-activated at or near the surface of a conductive electrode so that mass transport limitation may be encountered practically. Thus, immobilizing them into heterogeneous matrixes seems to be a promising way to tackle the above issues [110]. To this end, many conductive electrodes like carbon, indium tin oxide (ITO) and metals have been applied as substrates to load molecular complexes [111-114].

\section{MOFs AND COFs FOR ECR}

\section{MOFs and COFs with immobilized metal complexes}

As mentioned above, in spite of their high activity and selectivity for ECR, metal complexes are still not ideal for practical applications because of their low interfacial concentration and resulting inability to deliver high currents. Not to mention that they usually function in organic solvents and suffer from rapid deactivation. Thus, developing heterogeneous ECR catalysts based on molecular complexes has attracted much interest in recent years. In this part, we will mainly focus on recent work concerning immobilizing metal complexes into MOFs or COFs for ECR applications.

\section{Metalloporphyrin-based COFs}

Metalloporphyrins, possessing high thermal stability and approximate two-dimensional (2D) square geometry, have been demonstrated as feasible building blocks for many supramolecular architectures [115-117]. Those porous metalloporphyrin networks have exhibited potentials in many fields including sensor and size- and shape-selective catalysis $[118,119]$. With regard to ECR applications, it was until recent years that metalloporphyrin-based framework materials had been studied.

With the aim of creating extended planar $\pi$-electron systems that allow close intermolecular $\pi-\pi$ distances,
Yaghi and coworkers $[120,121]$ reported a 2D organic framework consisting of porphyrins (COF-366). Owing to its $\pi$-conjugated system and short interlayer distances, such material showed high charge carrier mobility and electrical conductivity. The porous structure of COF-366 was identified with a Brunauer-Emmett-Teller (BET) surface area of $1360 \mathrm{~m}^{2} \mathrm{~g}^{-1}$ and solution-accessible 1D channels of approximately $20 \AA$ in width (Fig. 3c). For the ECR test, the cobalt-metallized COF-366-Co was first evacuated by activation with supercritical carbon dioxide and deposited on the conductive carbon fabric. In neutral aqueous solutions (phosphate buffer, $\mathrm{pH} 7$ ), the COF366-Co electrode promoted the carbon monoxide evolution selectively with an FE of $90 \%, 10 \%$ higher than that of the molecular cobalt porphyrin system. At $-0.67 \mathrm{~V} v s$. RHE, which was in accordance with the $\mathrm{Co}(\mathrm{II}) / \mathrm{Co}(\mathrm{I})$ redox potential, the catalyst displayed optimal performances, consistent with former reports that the reduced $\mathrm{Co}(\mathrm{I})$-porphyrins are the active sites for $\mathrm{CO}_{2}$-to- $\mathrm{CO}$ reduction [122,123]. After continuous controlled potential electrolysis (CPE) for $24 \mathrm{~h}$, the COF catalyst, on a peractive-site basis, yielded a turnover number (TON) of 34,000 and an initial turnover frequency (TOF) of $2500 \mathrm{~h}^{-1}$, representing a substantial improvement over the molecular cobalt tetrakis( $N, N, N$ trimethyl-4-aminophenyl)porphyrin (Co(TAP)). Besides, it showed neither marked changes in crystallinity and microscopic morphology nor evidence of cobalt nanoparticle formation, revealing its superior stability for ECR in aqueous solutions.

Although the 2D MOF-366-Co resulting from co-facial stacks of porphyrins could act as conduits for the delivery of electrons from the underlying electrode to the many exposed Co sites, the narrow channels inside might hinder the adsorption of carbon dioxide, and thus only those metalloporphyrin centers sited at the termini of COF nanocrystals were accessible to molecular reactants, corresponding to an activity of $4 \%$ of cobalt sites.

Fortunately, owing to the possibility of multivariate synthesis offered by reticular chemistry, the topologically identical and functionally modified building blocks can be introduced into COF structures to tune their properties precisely. Through a modular reticular approach, they prepared the COF-367-Co analog using biphenyl-4,4'dicoarbonxaldehyde (BPDA) as the strut in place of 1,4benzenedicarboxaldehyde (BDA) (Fig. 3a). The extended COF-367-Co, possessing a higher BET surface area of $1470 \mathrm{~m}^{2} \mathrm{~g}^{-1}$ and a wider channel of $24 \AA$, exhibited an improved surface concentration of electroactive cobalt porphyrin sites over COF-366-Co, corresponding to ac- 

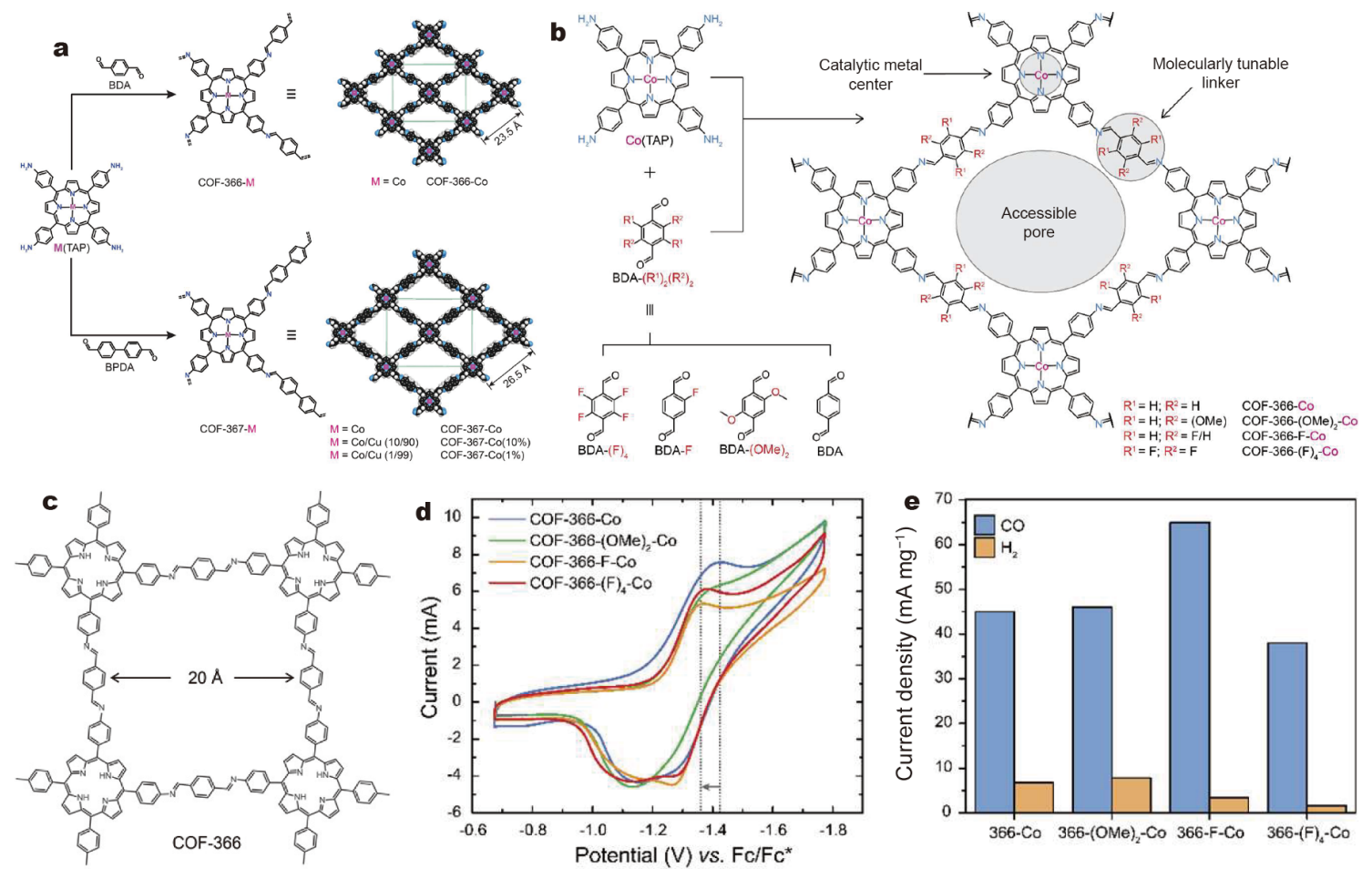

Figure 3 (a) Design and synthesis of COF-366-Co and COF-367-Co. Reprinted with permission from Ref. [121]. Copyright 2015, The American Association for the Advancement of Science (AAAS). (b) Systematic modulation of COF-366-Co through remote functionalization. Reprinted with permission from Ref. [125]. Copyright 2018, American Chemical Society. (c) Illustration of the pores in COF-366. Reprinted with permission from Ref. [120]. Copyright 2011, American Chemical Society. (d) Cyclic voltammograms (in DMF with tetrabutylammonium hexafluorophosphate as the electrolyte) and (e) current densities (at $-0.67 \mathrm{~V}$ vs. RHE in $0.5 \mathrm{~mol} \mathrm{~L}^{-1}$ aqueous potassium bicarbonate buffer) of modulated COFs [125].

cessibility of $8 \%$ of the cobalt sites in the entire framework. Consequently, such an extended COF showed enhanced performances for ECR with both smaller overpotential and higher catalytic current density. At $-0.67 \mathrm{~V} v s$. RHE, it yielded a TON of 48,000 with a high FE of $91 \%$.

Clearly, incorporating catalytic cobalt porphyrin units into COFs generated highly active, stable, and selective electrocatalysts for $\mathrm{CO}_{2}$-to- $\mathrm{CO}$ reduction in aqueous solutions. In addition to the steric effects of surface area and site isolation, the COF environment could directly modulate the electronic properties of molecular centers and furnish them with more delocalized electronic structures, thereby contributing to the observed gains in reaction selectivity and activity [124]. As introduced before, the reactivity of metal porphyrins can be tuned through introducing different substituent groups on the porphyrin rings. In the case of COFs consisting of metal porphyrins, owing to the efficient charge transport along the COF backbone, it is possible to optimize the electronic properties of the metal centers through proper remote functionalization of the linker units, and consequently to improve their reactivity.

Recently, based on the work of COF-366-Co, Yaghi and coworkers [125] reported a series of COFs with different electronegative elements substituted on the BDA units termed COF-366-(OMe) ${ }_{2}-\mathrm{Co}$, COF-366-F-Co and COF-

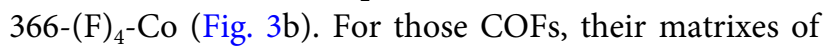
the structures remained essentially unaltered and their affinities to $\mathrm{CO}_{2}$ were similar, revealing that those factors hardly contributed to their different ECR reactivities. In contrast, since the proposed ECR mechanism with cobalt porphyrins suggested a first $\mathrm{Co}(\mathrm{II})$ reduction step to $\mathrm{Co}(\mathrm{I})$, the functionalization of electronegative elements could directly translate into an electron withdrawing on the cobalt centers and make them more prone to reduction, resulting in smaller overpotentials for CO generation. Consequently, when tested as electrode materials for ECR in organic electrolytes, the potential of the cathodic wave shifted from $-1.425 \mathrm{~V}$ vs. ferrocene/ferrocenium $\left(\mathrm{Fc} / \mathrm{Fc}^{+}\right)$for $\mathrm{COF}-366-\mathrm{Co}$ to $-1.380 \mathrm{~V}$ vs. $\mathrm{Fc} / \mathrm{Fc}^{+}$for $\mathrm{COF}-$ 366-F-Co (Fig. 3d). In neutral aqueous solutions, the electron withdrawing characteristics also improved the catalytic behavior of the framework with the current 
density for $\mathrm{CO}$ formation increased from $45 \mathrm{~mA} \mathrm{mg}^{-1}$ for COF-366-Co to $65 \mathrm{~mA} \mathrm{mg}^{-1}$ for COF-366-F-Co at $-0.67 \mathrm{~V}$ (vs. RHE) (Fig. 3e).

Accordingly, metal porphyrin-based COFs, possessing impressive features with high degree of electronic communication throughout the whole frameworks and organic backbones allowing for covalent modification, have demonstrated their potentials in supporting molecular catalysts for the electrochemical $\mathrm{CO}_{2}$-to- $\mathrm{CO}$ reduction. Meanwhile, fabricating more effective catalysts through reticular synthesis and exploring the reaction mechanisms through experiments and theoretical calculations are still highly required.

\section{Metalloporphyrin-based MOFs}

In addition to incorporating metal porphyrins into COFs through strong covalent bonds, constructing MOFs through strong metal-carboxyl bonds provides an alternative approach to immobilizing molecular catalysts into porous framework materials [126,127].

As discussed before, for heterogeneous systems, enhancement of either the molecular-scale reaction rate or the active-catalyst areal concentration might lead to greater catalytic current densities at a given applied potential. To this end, employing MOFs as high-surface area platforms for molecular catalysts should also be helpful to substantially boost their areal concentrations and lead to significant leaps in their catalytic performances. Besides, through covalent attachment to the framework nodes, the molecular catalysts could be loaded evenly in MOFs and separated physically, offering the opportunity to avoid aggregation and deactivation.

Hubb and coworkers [128] demonstrated the use of thin films of Fe-porphyrin-based MOF-525 as platforms for anchoring a substantial quantity of an electroactive molecular catalyst on a conductive electrode for ECR (Fig. 4a). Such MOF films possessed an ordered and porous heterogeneous network, permitting free permeation of electrolyte counterions and dissolved $\mathrm{CO}_{2}$ into the interior of the film. Meanwhile, following the mechanism of redox hopping between neighboring Fe-5,10,15,20-(4carboxyphenyl)porphyrin (TCPP) sites, they were able to transport electrons from the current collector towards the electrocatalytic sites (Fig. 4b) [129]. In detail, the electrophoretic deposited (EPD) Fe-MOF-525 film showed an electrocatalyst surface concentration of $6.2 \times 10^{-8} \mathrm{~mol} \mathrm{~cm}{ }^{-2}$, in comparison with $7 \times 10^{-11} \mathrm{~mol} \mathrm{~cm}^{-2}$ on a flat electrode (Fig. 4c), emphasizing the advantage of using the MOF as a strategy to boost the catalyst loading and consequently catalytic performance. When tested in acetonitrile/tetrabutylammonium hexafluorophosphate $\left(\mathrm{TBAPF}_{6}\right)$ solutions $\left(\mathrm{CO}_{2}\right.$-saturated), the Fe-MOF-525 system reduced $\mathrm{CO}_{2}$ to $\mathrm{CO}$ at current densities up to $2.3 \mathrm{~mA} \mathrm{~cm}^{-2}$ (FEs of $54 \% \pm 2 \%$ ), corresponding to a CO

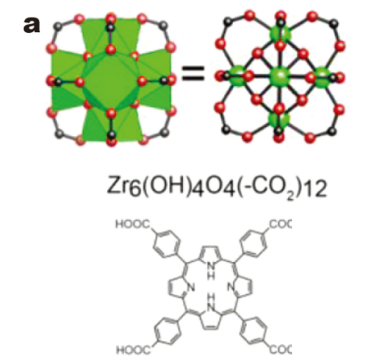

$\mathrm{H}_{4}-\mathrm{TCPP}-\mathrm{H}_{2}$

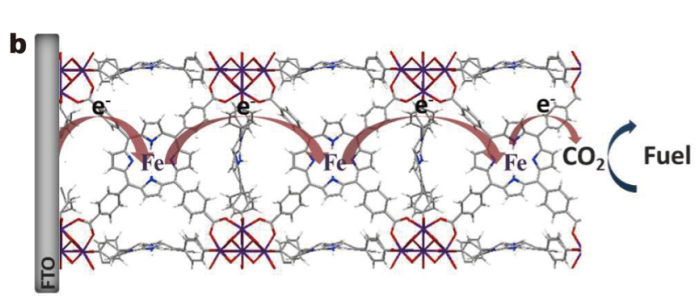

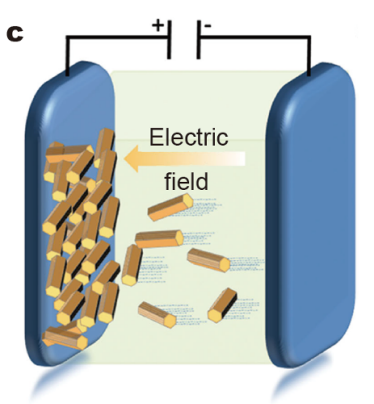

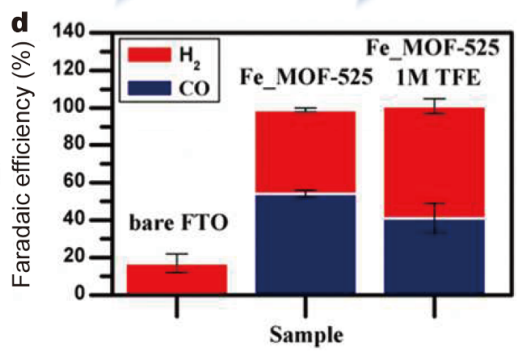

Figure 4 (a) Illustration of a portion of the crystal structure of MOF-525 in porphyrin free-base form, including the chemical structure of the TCPP linker and the $\mathrm{Zr}_{6}$-based node. Reprinted with permission from Ref. [128]. Copyright 2012, American Chemical Society. (b) Illustration of the surface structure of Fe-MOF-525 electrode, showing the mechanism of redox hopping between neighboring Fe-TCPP sites. Reprinted with permission from Ref. [129]. Copyright 2015, American Chemical Society. (c) Illustration of the principal for MOF EPD film growth. Reprinted with permission from Ref. [132]. Copyright 2014, Wiley-VCH. (d) FE of ECR on Fe-MOF-525 electrode [129]. 
TON of 272 and an average TOF of $64 \mathrm{~h}^{-1}$ (Fig. 4d). As demonstrated in the homogeneous Fe-TPP systems, the addition of weak Bronsted acids could elicit significant improvement in catalytic activity by favoring the protonation of the $\mathrm{Fe}-\mathrm{CO}_{2}$ adducts. Similarly, in the Fe-MOF525 system, the addition of $1 \mathrm{~mol} \mathrm{~L}^{-1}$ trifluoroethanol (TFE) increased the current densities up to $5.9 \mathrm{~mA} \mathrm{~cm}^{-2}$ (FEs of $41 \% \pm 8 \%$ for CO) and created a CO TON of 1520 and an average TOF of $468 \mathrm{~h}^{-1}$. The fact that appreciable catalytic current can be observed at potentials that are more positive than the $\mathrm{Fe}(\mathrm{I}) / \mathrm{Fe}(0)$ process implied that $\mathrm{Fe}$ (0)-TPP was highly active for $\mathrm{CO}_{2}$ reduction, in agreement with previous studies on Fe-TPP systems. Very recently, Dong and coworkers [130] reported another FeTCPP-based MOF of pocket channel frameworks (PCN)222(Fe) for ECR. Typically, the MOF was mixed with conductive additives (carbon black) in a ratio of $1: 2$ to improve its electric conductivity. When tested in $\mathrm{CO}_{2}{ }^{-}$ saturated aqueous solutions, the composite $\mathrm{PCN}$ $222(\mathrm{Fe}) / \mathrm{C}$ showed selective $\mathrm{CO}_{2}$-to- $\mathrm{CO}$ reduction with a current density of $1.2 \mathrm{~mA} \mathrm{~cm}^{-2}$ and an FE of $91 \%$. A TOF of $0.336 \mathrm{~s}^{-1}$ was estimated based on the surface concentration of active sites $\left(1.622 \times 10^{-8} \mathrm{~mol} \mathrm{~cm}^{-2}\right)$.

Yang and coworkers [131] demonstrated an atomic layer deposition (ALD)-assisted strategy to fabricate Coporphyrin based MOF thin films for ECR, in which MOFs grew in situ on the conductive substrate. Typically, aluminum oxide thin films were deposited onto the conductive substrate as metal precursors and subsequently reacted with cobalt porphyrin to form MOF films via a dissolution-recrystallization mechanism (Fig. 5). Growing porous thin MOF films on the conductive support guaranteed that each site was simultaneously exposed to the electrolyte and electrically connected to the electrode, resulting in high concentration of active sites (up to $1.1 \times 10^{-7} \mathrm{~mol} \mathrm{~cm}^{-2}$ ). Besides, compared with post-synthetic depositions, the ALD-based MOF conver- sion technique allowed precise control on catalyst loading, enabling the optimization to balance the active-site density with mass/charge transfer [132]. In aqueous solutions, the optimized Co-porphyrin based MOF thin films exhibited selective $\mathrm{CO}_{2}$-to-CO reduction with an $\mathrm{FE}$ of $76 \%$ at $-0.7 \mathrm{~V}$ vs. RHE and sustained a steady electrocatalysis over $7 \mathrm{~h}$ with a TON of 1400 per-site. They also proved that the majority of catalytic sites in this MOF were cobalt centers and the $\mathrm{Co}(\mathrm{I})$ species were critical to form Co- $\mathrm{CO}_{2}$ adducts, in accordance with many former reports on molecular cobalt porphyrins $[133,134]$.

Accordingly, using MOFs as heterogeneous platforms represents an efficient strategy to reticulate catalytic molecular units into a porous network with concentrated active sites, enabling the MOFs to provide higher catalytic currents. However, the rates of catalytic reduction of $\mathrm{CO}_{2}$ by the MOF films were still largely limited by the sluggish charge transfer, leading to small TOF values relative to those of the homogenous catalyst. Therefore, boosting charge diffusion rates will be necessary for fully realizing their promises as electrocatalysts.

\section{Other molecular complex-based MOFs}

In addition to metal porphyrins, other molecular catalysts have also been incorporated into MOFs to produce ECR catalysts. For example, Sun and coworkers [135] fabricated an [001] oriented Re-MOF film on a conductive fluourine-doped tin oxide (FTO) electrode via liquidphase epitaxy (LPE) (Fig. 6a). Theoretical calculation revealed that the oriented growth along [001] direction of Re-MOF favored the charge transfer mobility and contributed to the highly favorable electrocatalytic performances (Fig. 6b). When tested as the electrode for ECR in an organic electrolyte, the Re-MOF film followed the features of related molecular catalysts with two reduction peaks centered at -0.7 and $-0.9 \mathrm{~V} v s$. normal hydrogen electrode (NHE), corresponding to the reduction of bi-
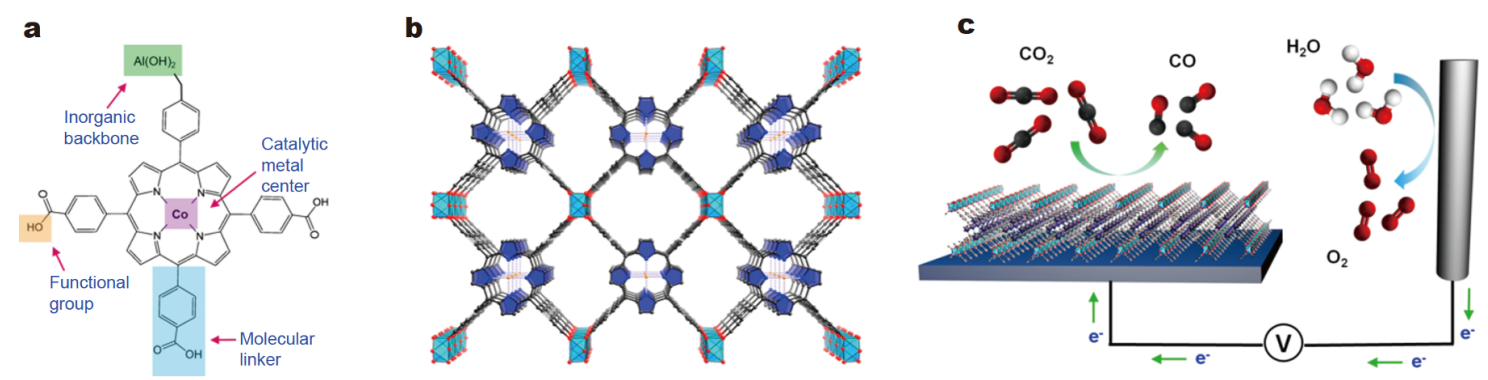

Figure 5 (a) Co-metalated TCPP units. (b) Illustration of the 3D MOF assembly. Co, orange spheres; O, red spheres; C, black spheres; N, blue spheres; Al, light-blue octahedra; and pyrrole ring, blue. (c) Functional principles of ECR in the integrated system. Reprinted with permission from Ref. [131]. Copyright 2015, American Chemical Society. 
a

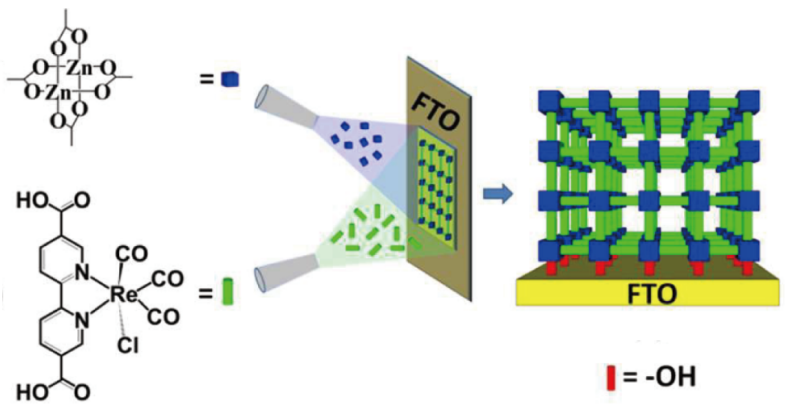

b

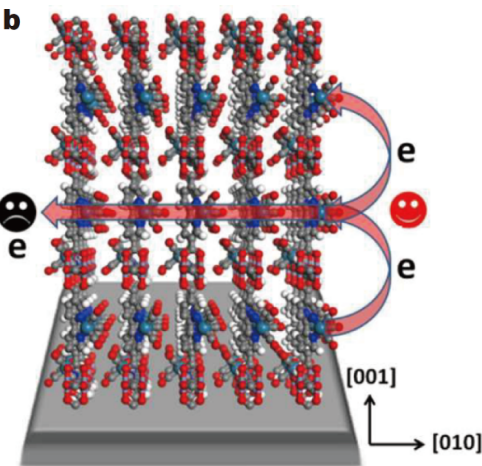

C

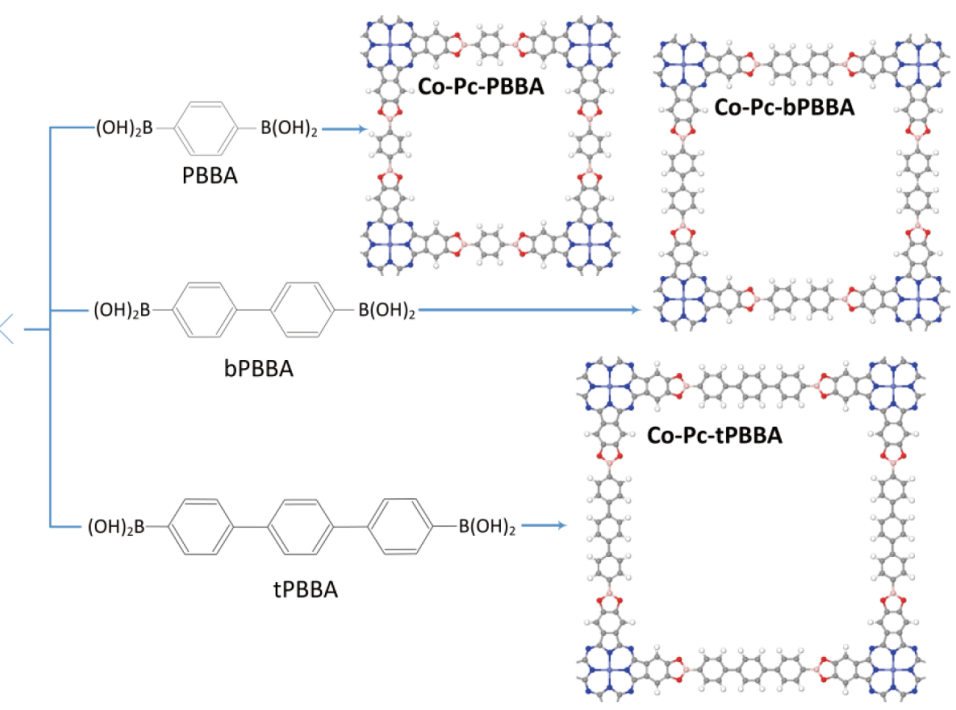

Figure 6 (a) Schematic representation of the fabrication process of Re-MOF on the functionalized FTO substrate in a layer-by-layer fashion. (b) Illustration of the preferred charge transfer pathway in epitaxial Re-MOF along the [001] direction. Reprinted with permission from Ref. [135]. Copyright 2016, The Royal Society of Chemistry. (c) Proposed structures of the cobalt phthalocyanine-based COF series. Reprinted with permission from Ref. [137]. Copyright 2018, Wiley-VCH.

pyridyl and $\operatorname{Re}(\mathrm{I})$, respectively. At $-1.6 \mathrm{~V}$ vs. NHE, it exhibited high selectivity in $\mathrm{CO}_{2}$-to- $\mathrm{CO}$ reduction with an $\mathrm{FE}$ of $93 \% \pm 5 \%$ and current densities exceeding $2.5 \mathrm{~mA} \mathrm{~cm}^{-2}$, considerably outperforming the free Rebipyridine complexes [136]. Such a high catalytic activity was attributed to its hierarchical structures loading more electroactive moieties (estimated to be $7 \times 10^{-8} \mathrm{~mol} \mathrm{~cm}^{-2}$ ), giving a TON of 580 and an initial TOF of $690 \mathrm{~h}^{-1}$. However, the current density gradually decreased with continuous electrolysis for $0.5 \mathrm{~h}$, indicating the degradation of Re-MOF. Hence, the stability of this system needs to be improved for further applications.

Recently, Jiang and coworkers [137] provided a prototype of incorporating cobalt phthalocyanine into a new COF series through covalent bonds linking to boronic acid and predicted them to be promising catalysts for ECR (Fig. 6c). In detail, first principle and classical molecular dynamic calculations revealed that the energy level of Co 3d orbital in Co-phthalocyanine was higher than that in Co-porphyrin, endowing such COF series with higher activities in adsorbing reaction intermediates and lower energy barriers for their reductions. Although further experiments are still needed, this case indicates that advanced computational sciences have already shown their potentials in guiding material synthesis and will play more and more important roles in conducting scientific researches $[138,139]$.

\section{MOFs without metal complexes}

As discussed in the former part of this section, MOFs and COFs have been demonstrated to be reliable platforms to immobilize molecular catalysts. However, in those systems, the products were mainly in gas phases because those incorporated metal complexes usually favored $\mathrm{CO}_{2}-$ to-CO reduction. In the following part, some MOFs without molecular catalyst units and their performances 
in catalyzing ECR will be introduced.

\section{Traditional MOF-based materials}

Among metallic catalysts, $\mathrm{Cu}$ uniquely produces hydrocarbons at high reaction rates over sustained periods of time, whereas it usually generates a range of reaction products [140-142]. Thus, fabricating Cu-based catalysts possessing proper surficial structures and intrinsic properties is considered to be applicable for selective and active $\mathrm{CO}_{2}$ reduction. To this end, constructing $\mathrm{Cu}$-based MOFs has attracted more attentions because of their combined favorable properties of large surface area, tunable pore-size, shape-selective characters and electronic conductivities.

In 2012, Hinogami et al. [143] reported the first trial to employ Cu-based MOF (CR-MOF) in the electrochemical reduction of $\mathrm{CO}_{2}$. Owing to the adsorption of protons to nitrogen atoms in the ligands and the accompanying electron transfer associated with $\mathrm{Cu}(\mathrm{II})$ ions, the CRMOF had been demonstrated to be both electron and proton conductive, enabling the dispersed metal sites to be accessible to the readily supply of electrons and protons. Those advantages in combination with the nanopores restricting the reaction pathway contributed to the high activity and selectivity of CR-MOF in ECR. In $\mathrm{CO}_{2}-$ saturated aqueous solution, the CR-MOF electrode showed a more positive onset potential by about $0.2 \mathrm{~V}$ than that of the $\mathrm{Cu}$ metal electrode, indicating its better catalytic activity for $\mathrm{CO}_{2}$ reduction. Besides, it reduced $\mathrm{CO}_{2}$ to $\mathrm{HCOOH}$ with a selectivity of more than $98 \%$ and exhibited a stable catalytic performance, whereas $\mathrm{Cu}$ electrode generated a range of products and underwent rapid decrease in catalytic activity.

Later in the same year, Kulandainathan and coworkers [144] investigated another Cu-based MOF (widely known as HKUST-1) for ECR. When tested in $\mathrm{CO}_{2}$-saturated $\mathrm{DMF} /$ tetrabutylammonium tetrafluoroborate (TBATFB) solutions, the MOF-coated glassy carbon (GC) electrode displayed a smaller overpotential and a higher catalytic current density for $\mathrm{CO}_{2}$ reduction than those of a bare GC electrode, bringing out its electrocatalytic activity on ECR. CPE revealed that the major product was oxalic acid with an FE of 51\%, and such relatively high selectivity was attributed to the $\mathrm{Cu}(\mathrm{I})$ sites being able to stabilize carbon dioxide radical anions which further dimerized into oxalate anions [145].

Albo et al. [146] further investigated the HKUST-1 for ECR in aqueous solutions by fabricating gas diffusion electrodes (GDEs), which are widely employed to increase the $\mathrm{CO}_{2}$ concentration at or near the catalytic sites and to facilitate mass transports. More details about the GDE design will be discussed later [146]. Under optimized conditions, the HKUST-1 GDE reduced $\mathrm{CO}_{2}$ to liquid alcohols with $\mathrm{CH}_{3} \mathrm{OH}$ and $\mathrm{C}_{2} \mathrm{H}_{5} \mathrm{OH}$ as the predominant products in a total $\mathrm{FE}$ of $15.9 \%$. In this system, those unsaturated metal sites exposed in the porous frameworks were prone to interact strongly with reaction intermediates and favored the $\mathrm{C}-\mathrm{C}$ coupling reactions. However, further computational and experimental efforts are needed to fully elucidate the $\mathrm{CO}_{2}$ reduction elementary steps. Activity degradation with dropped FEs happened inevitably in long-term electrocatalysis. Experimental results revealed that the MOF first transformed into amorphous phases and further underwent generation to $\mathrm{Cu}$ (II) hydroxycarbonate partially, indicating that the HKUST-1 was not stable in water and might undergo hydrolysis or other reaction causing the decrease of active sites. Those drawbacks in addition to the leaching effect of the GDE were plausible reasons for its decrease in electrocatalytic performances [147]. Interestingly, the amorphous HKUST-1 still exhibited competitive property in reducing $\mathrm{CO}_{2}$ to alcohols, which gave us a clue that employing materials with low crystallinity might also be viable once they possess suitable geometric and electronic structures.

Recently, Irigaray and coworkers [148] tried to introduce heteroatoms $(\mathrm{Zn}(\mathrm{II}), \mathrm{Ru}(\mathrm{III})$ and $\mathrm{Pd}(\mathrm{II}))$ to the benchmark HKUST-1 and investigated their effects on electrocatalytic $\mathrm{CO}_{2}$ reduction. Consequently, all of those samples showed structures similar to the pristine HKUST-1 except for enlarged cell parameters, caused by the occurrence of longer metal-oxygen bonds. Loaded on GDEs and tested in aqueous solutions, the $\mathrm{Zn}$ (II)-doped sample showed a similar ECR trend with the pristine MOF, while the incorporation of $\mathrm{Pd}(\mathrm{II})$ hindered the electroreduction process of $\mathrm{CO}_{2}$ to liquid products and promoted $\mathrm{H}_{2}$ revolution. Interestingly, the $\mathrm{Ru}(\mathrm{III})$-doped sample appeared to be the most promising candidate with an $\mathrm{FE}$ of $47.2 \%$ in reducing $\mathrm{CO}_{2}$ to alcohols (majorly $\mathrm{CH}_{3} \mathrm{OH}$ and $\mathrm{C}_{2} \mathrm{H}_{5} \mathrm{OH}$ ). In addition, increasing the dopant loading could promote the ethanol selectivity reaching values up to $100 \%$, which was attributed to the stronger interactions between the dopant metals and reaction intermediates that favored $\mathrm{C}-\mathrm{C}$ coupling reactions $[149,150]$. However, its activity fell abruptly to plateau close to an FE of $10 \%$ and impurities were also detected after long-term running.

Kang and coworkers [151] further replaced $\mathrm{Cu}$ by $\mathrm{Zn}$ to produce a pure Zn-based MOF and investigated its performances as the electrocatalyst for ECR (Fig. 7). When 
deposited on carbon paper (CP) and tested in ILs, such $\mathrm{Zn}$-based MOF/CP electrode showed superior capabilities in reducing $\mathrm{CO}_{2}$ to $\mathrm{CH}_{4}$ selectively. In an optimized sample with the largest electrochemical surface areas and lowest charge transfer resistance $\left(R_{\mathrm{ct}}\right)$, the selectivity of $\mathrm{CH}_{4}$ could be higher than $80 \%$ at a current density of higher than $3 \mathrm{~mA} \mathrm{~cm}^{-2}$ with an overpotential of $0.25 \mathrm{~V}$. In this system, both electrode materials and electrolytes were demonstrated to be crucial for its high activity and selectivity (Fig. 7e). Firstly, the $\mathrm{Zn}-\mathrm{MOF}$ displayed stronger adsorption for $\mathrm{CO}$ than for $\mathrm{CH}_{4}$, promoting the generated $\mathrm{CO}$ molecules to be further reduced. Besides, the $\mathrm{Zn}-\mathrm{MOF}$ s were synthesized in ILs which endowed them with good compatibility with electrolytes and favored the mass transports [152]. Moreover, the imidazolium-based ILs containing fluorine could adsorb and activate $\mathrm{CO}_{2}$, which in combination with the large MOF surfaces accommodating ILs benefited the high electrochemical activity of this system (Fig. 7f, g).

\section{Zeolitic imidazolate framework (ZIF)-based materials}

ZIF, which is a subclass of MOFs and combines highly desirable properties of zeolites and MOFs, has emerged as a novel type of crystalline porous materials for versatile applications [153-155]. Specially, many ZIFs with stable structures could be prepared under moderate conditions in water, making them suitable for electrochemical researches in aqueous solutions [156-158]. Wang and coworkers [159] reported the first investigation in employing ZIF for ECR. In aqueous solutions, the Zn-based ZIF (ZIF-8) electrode could deliver a high catalytic ac- tivity towards $\mathrm{CO}_{2}$ electroreduction as well as a decent stability with $60 \%$ CO yield lasting for more than $4 \mathrm{~h}$. In controlled experiments, neither the ZIF-67 (a Co-based ZIF with similar structures with ZIF-8) nor the decomposed ZIF-8 showed comparable $\mathrm{CO}_{2}$ reduction reactivity, so that the discrete $\mathrm{Zn}$ nodes undergoing reversible redox reactions were thought to be critical for high CO selectivity. Meanwhile, the counter anions in electrolytes were proved to have intriguing effects on electrocatalysis because suitable anions being able to easily adsorb into and desorb from the ZIF pores could allow facile anion exchanges for charge balance and lead to the enhanced electrochemical reduction of $\mathrm{CO}_{2}[160]$.

\section{Other functions of MOFs and COFs in ECR}

In above systems of MOFs and COFs, whether with or without molecular catalyst units, metal centers were regarded as the electrocatalytic sites towards $\mathrm{CO}_{2}$ reduction. In the following part, we will introduce some other roles that framework materials might play in ECR other than providing active centers.

\section{Supporting catalytic species}

Commonly, the ultrahigh specific surface areas and periodic intraframework chemical functionality of MOFs create the possibility for installing well-separated active sites within the framework with extremely high active-site density. Those features also render MOFs appealing as ideal support for immobilization of other functional materials. Thus, embedding nanoparticles within MOFs has been proved to be applicable in fabricating advanced

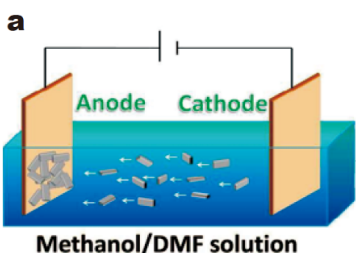

Methanol/DMF solution

$\mathbf{e}$

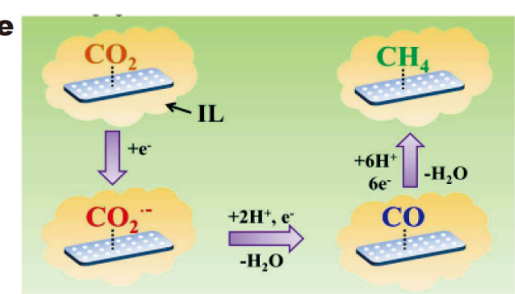

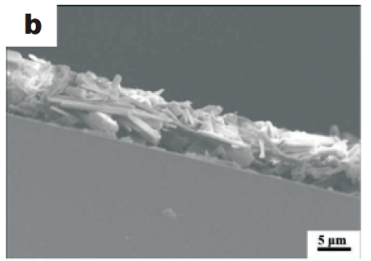
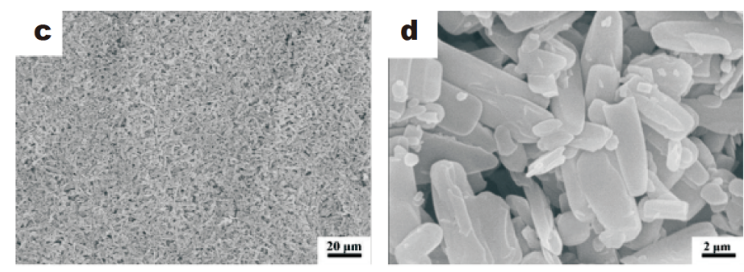

f

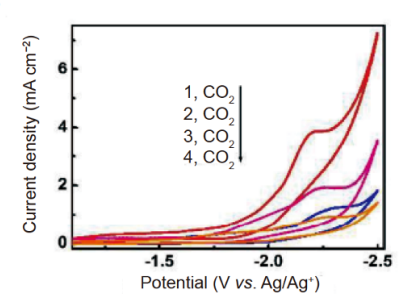

$\mathbf{g}$

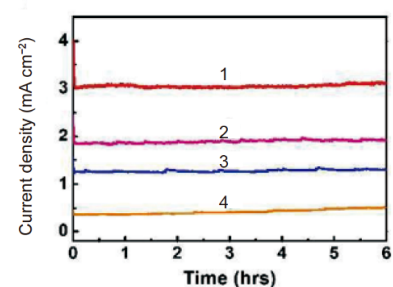

Figure 7 (a) Schematic fabrication of the Zn-based MOF electrode. Scanning electron microscopy (SEM) images of (b) the cross section of the electrode, (c) electrode surface and (d) the amplified electrode surface. (e) The possible pathway for electrochemical reduction of $\mathrm{CO}_{2}$ to $\mathrm{CH}_{4}$ on the Zn-MOF/CP cathode in ILs. (f) Cyclic voltammetry (CV) curves and (g) current density profiles (at $-2.2 \mathrm{~V} v s . \mathrm{Ag} / \mathrm{Ag}^{+}$) in different ILs: 1 -BmimBF $; 2$ BmimOTf; 3-BmimPF 6 ; 4-BmimClO 4 . Reprinted with permission from Ref. [151]. Copyright 2015, The Royal Society of Chemistry. 
catalysts [161-163].

Kung et al. [164] demonstrated that such strategy was also viable to produce functional electrocatalysts for ECR. $V i a$ an approach termed solution-phase analogue of ALD in MOFs, $\mathrm{Cu}(\mathrm{II})$ clusters were installed in a zirconiumbased MOF (NU-1000) uniformly (Fig. 8). Although a large amount of $\mathrm{Cu}(\mathrm{II})$ sites were introduced in the $\mathrm{Cu}$ (II)- NU-1000, only a few of them were electrochemically addressable due to the poor conductivity of NU-1000 [165]. However, further electroreduction detaching $\mathrm{Cu}$ from the NU-1000 framework produced $\mathrm{Cu}$ nanoparticles (smaller than $10 \mathrm{~nm}$ ) inside the MOF channels and largely increased the fraction of electroactive $\mathrm{Cu}$ sites from $0.5 \%$ to $16 \%$ of the total amount of $\mathrm{Cu}$. Consequently, in a $\mathrm{CO}_{2}$-saturated aqueous electrolyte solution containing $0.1 \mathrm{~mol} \mathrm{~L}^{-1} \mathrm{NaClO}_{4}$, the $\mathrm{Cu}-\mathrm{NU}-1000$ could effectively reduce $\mathrm{CO}_{2}$ to $\mathrm{HCOOH}$ and $\mathrm{CO}$ at a current density of about $1.2 \mathrm{~mA} \mathrm{~cm}^{-2}(-0.82 \mathrm{~V}$ vs. RHE) and an FE of $31 \%$, which was broadly consistent with results reported previously for ECR on $\mathrm{Cu}$ electrodes. In spite of their competitive reactivity toward ECR, employing MOFs to support electrocatalysts for ECR constitutes a nascent subfield and more efforts should be paid to improve their selectivity and activity.

\section{Capturing $\mathrm{CO}_{2}$}

One of the long-standing issues that prohibit large-scale $\mathrm{CO}_{2}$ electroreduction is the suppressed reaction rate, which is correlated with the limited reaction areas of bulk electrodes and poor solubility of $\mathrm{CO}_{2}$ in most solvents. To solve this problem, embedding catalysts on GDEs has been widely utilized [166-168]. Generally, GDEs are electroconductive composites constructed on porous materials, and they have the advantage of establishing stable and extended three-phase boundaries (TPB) of gasliquid-solid interface, allowing sufficient exposure of catalysts to the reactants as well as fast exchange of reactants and products. Nonetheless, the reduction efficiency and product selectivity of ECR are still limited by the low $\mathrm{CO}_{2}$ solubility in aqueous solutions [169]. Therefore, it is necessary to find materials/designs that can remarkably increase local $\mathrm{CO}_{2}$ concentration on the basis of contemporary GDEs. Actually, MOFs and COFs should be suitable candidates because they have thoroughly demonstrated strengths for gas storage and separation (Fig. 9a) [170-172]. Moreover, some of them could adsorb and release large amount of $\mathrm{CO}_{2}$ under room temperature reversibly, which will favor smooth $\mathrm{CO}_{2}$ transport and the consequent reduction (Fig. 9b) [173-177].

To this end, Qiu and coworkers [178] introduced the $\mathrm{Cu}$-based MOF into $\mathrm{Cu}$ nanoparticles decorated GEDs and demonstrated that it could efficiently lower the onset potential and improve the selectivity of $\mathrm{CH}_{4}$ in ECR. Functioning as the $\mathrm{CO}_{2}$ capturer, the $\mathrm{Cu}$-based MOF could increase the $\mathrm{CO}_{2}$ concertation on the interface
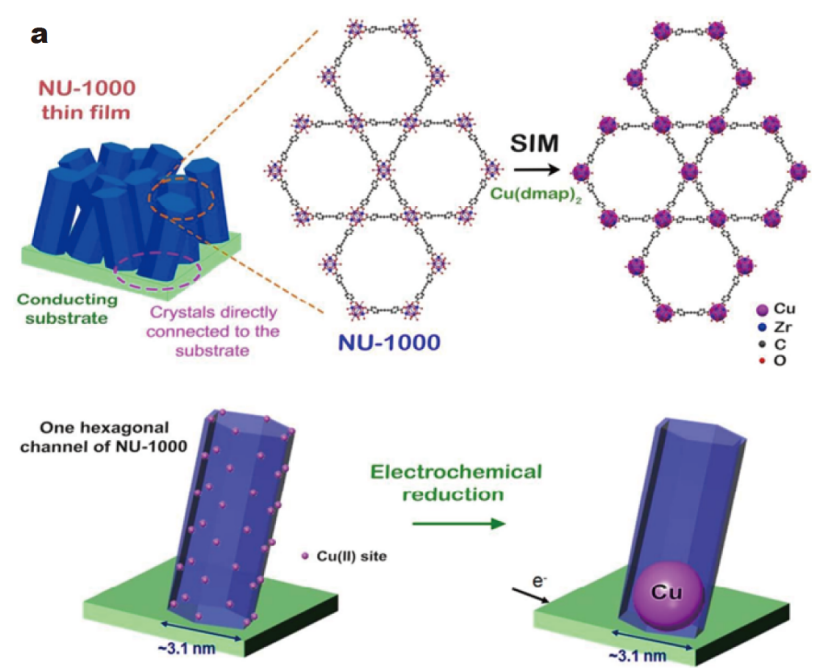
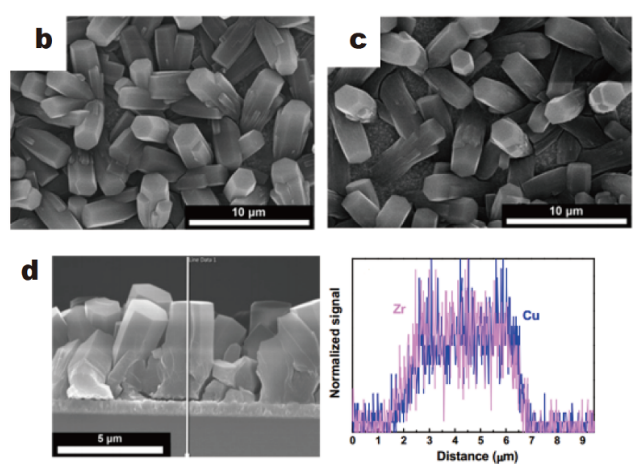

e

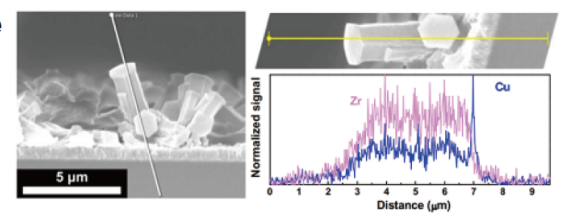

Figure 8 (a) Schematic representation of solvothermal-deposition in MOFs (SIM) to install single-site Cu(II) into the NU-1000 thin film and the electrochemical reduction of $\mathrm{Cu}(\mathrm{II})$ to generate metallic Cu nanoparticles. Top-view SEM images of the (b) NU-1000 thin film and (c) Cu-SIM NU1000 thin film. (d) Cross-section SEM image of the Cu-SIM NU-1000 thin film (left part) and energy dispersive X-ray spectroscopy (EDS) line scan results (right part). (e) Cross-section SEM image of the reduced Cu-SIM NU-1000 thin film (left part) and EDS line scan results (right part). Reprinted with permission from Ref. [164]. Copyright 2017, American Chemical Society. 


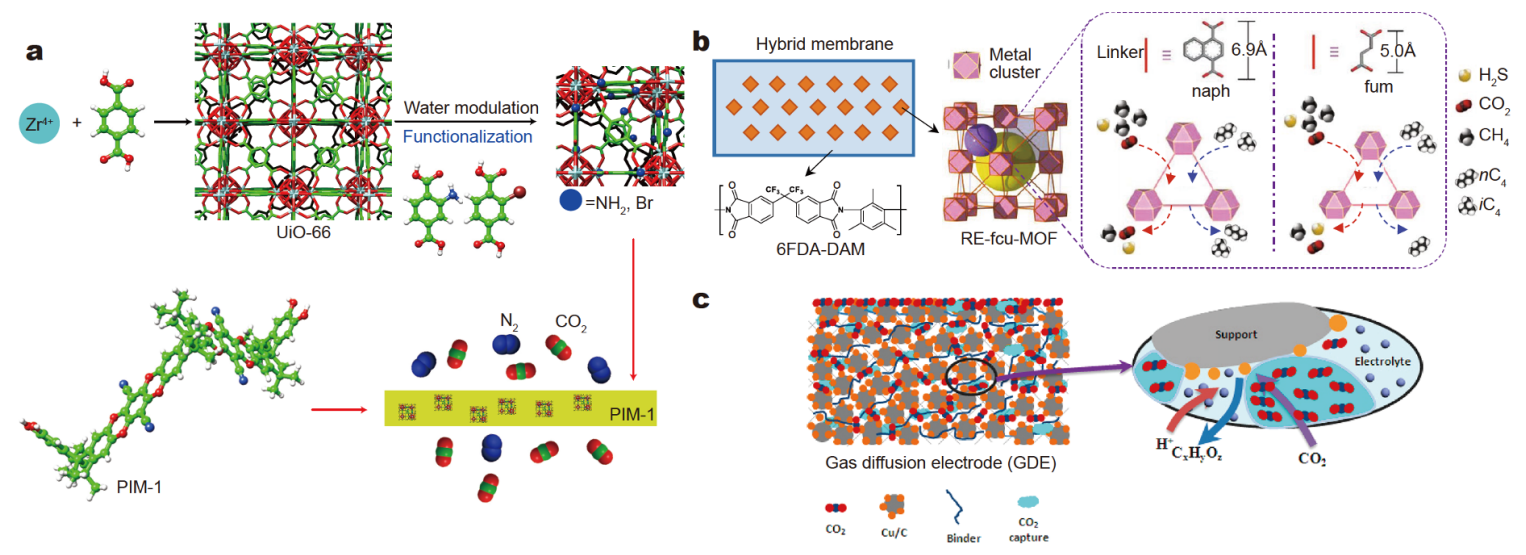

Figure 9 (a) Schematic representation of the functionalized MOF (UiO-66) for $\mathrm{CO}_{2}$ separation. Reprinted with permission from Ref. [176]. Copyright 2017, Springer Nature Publishing Group. (b) Flexible design on MOF pores by varying the length of linkers for gas separation. Reprinted with permission from Ref. [171]. Copyright 2018, Springer Nature Publishing Group. (c) Structural schematic diagram of GDE with Cu-based MOF as $\mathrm{CO}_{2}$ capturer. Reprinted with permission from Ref. [178]. Copyright 2017, American Chemical Society.

between GDE and the electrolyte (Fig. 9c), thereby facilitating $\mathrm{CO}_{2}$ transport and inhibiting HER. Specifically, in an optimized system, the modified electrode delivered $\mathrm{FE}$ of $\mathrm{CH}_{4} 2-3$ folds higher than that of the GDE without $\mathrm{Cu}-\mathrm{MOF}$ capturer and suppressed HER remarkably with FE of $\mathrm{H}_{2}$ decreasing up to $30 \%$. However, the introduction of $\mathrm{Cu}$-based MOF to GDE also induced negative effect, regarding to the insulating effect to block the electron transfer. Thus, the ECR reactivity was mitigated by the competing results of the beneficial effect of the $\mathrm{CO}_{2}$ concentration improvement and the blocking effect on the $\mathrm{Cu}$ active sites. Although the modified electrode could perform stable catalytic activity and selectivity for $6 \mathrm{~h}$, structure and performance degradation were inevitable in prolonged time-period because the MOF was not stable in water.

Inspired by their work, we speculate that integrating the storage and catalytic units together should be promising and the emerging reticular chemistry could provide applicable tools to fabricate framework materials combining the capturing and active sites, providing unparalleled opportunities for exploring effective electrocatalysts for ECR. With the integrated design, the $\mathrm{CO}_{2}$ concentration around the reduction centers will be much higher than that in the bulk solution, resulting in the smaller overpotential. Besides, according to the chemical collision theory, the promoting effect of high reactant concentration would be even more remarkable from the kinetic aspect, if all $\mathrm{CO}_{2}$ are electrochemically active [179]. Generally, in aqueous solutions where protons transfer is fast, increasing $\mathrm{CO}_{2}$ concentration can greatly enhance the reaction rate.

\section{Modifying electrode surfaces}

Commonly, many MOFs and COFs could uptake a large amount of $\mathrm{CO}_{2}$ through physisorption and the weak interaction has been thought to be critical for quick adsorption-desorption cycles. Very recently, Liu et al. [180] reported that amine-based COFs being able to chemically adsorb $\mathrm{CO}_{2}$ molecules are effective coatings to improve the selectivity of traditional silver metal electrode in catalyzing ECR.

Typically, the amine-based COFs were produced through direct reduction of their parent imine-linked frameworks (COF-300 and COF-366-M, where $\mathrm{M}=\mathrm{Co}$, $\mathrm{Cu}$ and $\mathrm{Zn}$ ) (Fig. 10a). In those reduced COFs, the crystallinity and underlying topology were fully retained, while the newly formed amine linkages provided exceptional chemical stability in strong aqueous acid and base. Besides, the abundant amino groups could act as chemisorptive sites to selectively capture $\mathrm{CO}_{2}$ molecules by forming carbamate (Fig. 10d), which was believed to be the key transition state for $\mathrm{CO}_{2}$-to- $\mathrm{CO}$ reduction in this system. When deposited on a silver electrode for ECR, the excellent chemical stability of these COFs in combination with the amine functional groups led to a robust and molecularly defined interface at the silver metal surface (Fig. 10b, c). Consequently, the concerted operation of COFs and the Ag metal surface provided high conversion efficiency and excellent selectivity of ECR against HER. Typically, the CO conversion efficiency was raised from $43 \%$ to $80 \%$ and that for HER was suppressed to $9 \%$ at $-0.85 \mathrm{~V} v s$. RHE (Fig. 10e, f). Accordingly, constructing concerted electrode with framework materials and traditional metals represents a promising direction for acces- 

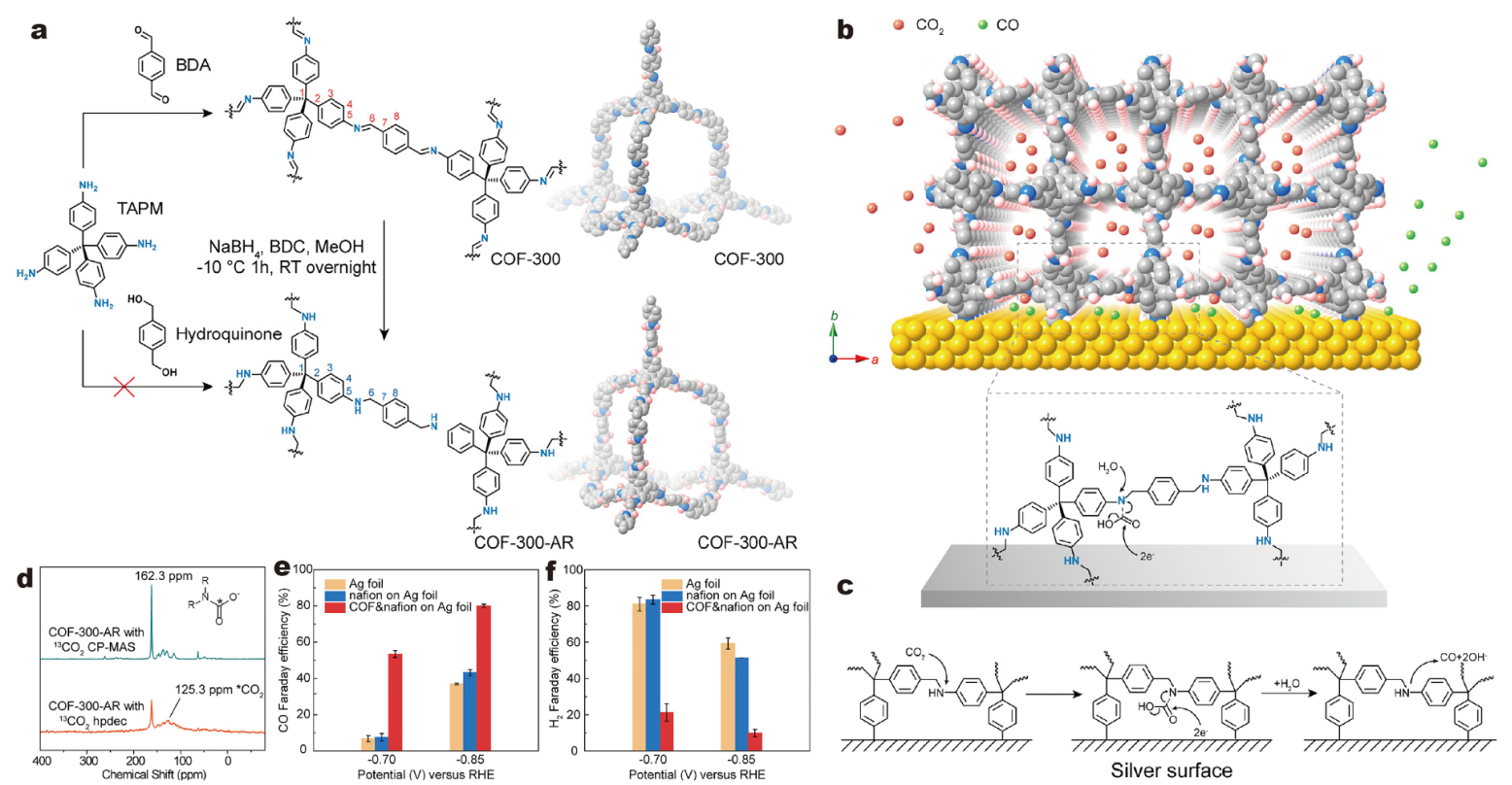

Figure 10 (a) Scheme for COF-300 reduction. In the space-filling diagrams, carbon and nitrogen atoms are represented as gray and blue spheres, respectively. Only the hydrogen atoms on the imine and amine linkage are shown (in pink) for clarity. (b) Illustration of the molecularly defined interface created by COF-300-AR on a flat silver electrode. (c) Scheme of the mechanism of concerted $\mathrm{CO}_{2}$ reduction taking place at the interface. (d) ${ }^{13} \mathrm{C}$ cross polarization magic angle spinning nuclear magnetic resonance (CP-MAS NMR) results showing the formation of carbamate intermediate during $\mathrm{CO}_{2}$ reduction process. FEs for (e) $\mathrm{CO}$ and (f) $\mathrm{H}_{2}$ production on the concerted electrode. Reprinted with permission from Ref. [180]. Copyright 2018, Cell Press.

sing high efficiency and selectivity in ECR.

\section{Short summary}

In short, combining the merits of heterogeneous and homogeneous systems, MOFs and COFs have demonstrated their potentials in immobilizing molecular catalysts as well as functioning as versatile electrocatalysts or electrocatalytic promoters for ECR. Generally, unique characteristics of framework materials contribute to their outstanding performances in catalyzing ECR. Firstly, the dispersion and isolation of actives sites could maximize their utilization especially with the help of advanced nanoengineering technologies of fabricating MOFs or COFs thin films on conductive substrates, which in combination with the high porosity and surface areas greatly facilitate the access of reactants to catalytic sites. In addition, those interconnected frameworks could adsorb $\mathrm{CO}_{2}$ molecules physically or chemically and improve their local concentration at the electrode-electrolyte interfaces, thereby increasing the rate of ECR and suppressing HER. Besides, owing to their modulative nature, those framework materials could be modified by grafting desired groups onto their metal centers or organic linkers as well as by tuning their interconnected pores to allow smooth molecule transportation. In this sense, multiple functions could be fulfilled in a single MOF- or COF-based system, further benefiting the catalytic reactivity. Moreover, the well-defined electronic and geometric structures in MOFs or COFs are beneficial to deduce the relationship between structure and property, shedding light on understanding their reaction mechanisms.

Though tremendous advances have been achieved, employing MOFs or COFs in catalyzing ECR is still in its infancy and several drawbacks should be improved in future research. Firstly, most of those MOFs or COFs are not ideal electronic conductors and the limited charge carrier mobilities impede them from delivering their full potentials, resulting in low catalytic current densities and large overpotentials. Besides, most of them are not stable in aqueous solutions, leading to inevitable performance degradations in long-term electrocatalysis. Fortunately, with the help of potent tools provided by the emerging reticular chemistry, improving water-stability and engendering electroconductivity in MOFs or COFs have been proved to be achievable.

\section{MOFs- AND COFs-DERIVED MATERIALS FOR ECR}

Framework materials, containing both inorganic metal centers and organic ligands, can serve as ideal sacrificial 
precursors to afford highly dispersed metal/metallic compound/carbon materials or their composites via facile calcination-thermolysis strategies [181-185]. Thus, MOFand COF-derived materials have also attracted numerous attentions for they to some extent inherit advantages of their parent materials and provide enhanced conductivity and stability. In this part, we introduce some of those materials explored for ECR and discuss their strengths and weaknesses.

\section{MOFs- and COFs-derived carbon materials}

As mentioned before, doping heteroatoms (e.g., N, B and P) into carbon structures has been widely explored to improve the chemical, electrical, and functional properties of carbon materials for ECR. Actually, framework materials containing heteroatoms should be ideal sacrificial templates for the synthesis of heteroatom-doped porous carbons because of their high porosity and evenly dispersion of atoms [186-188]. For instance, Wang and coworkers [189] reported a nitrogen-doped carbon through direct pyrolysis of ZIF-8 (Fig. 11a, b), followed by a subsequent acid treatment, and demonstrated it to be an effective electrocatalyst for $\mathrm{CO}_{2}$-to- $\mathrm{CO}$ reduction. Specifically, when applied as an electrode material in aqueous solutions, the $\mathrm{N}$-doped carbon exhibited a stable current density of $1.1 \mathrm{~mA} \mathrm{~cm}^{-2}$ and an $\mathrm{FE}$ of $~ 80 \%$ for $\mathrm{CO}$ at $-0.93 \mathrm{~V}$ vs. RHE (Fig. 11c). The high activity and selectivity were attributed to the presence of large amount of functional $\mathrm{N}$ species in the carbon structures for they could facilitate the transfer of proton-electron pairs to $\mathrm{CO}_{2}$, thereby lowering the energy barrier for the production of $* \mathrm{COOH}$, an intermediate for $\mathrm{CO}$ formation. In addition, the well-developed porosity further improved the activity by making more active sites accessible. However, the direct pyrolysis of framework materials usually leads to drastic decrease of specific surface areas, which is attributed to the collapse of the well-defined microporous structures $[190,191]$. Besides, those dis- a

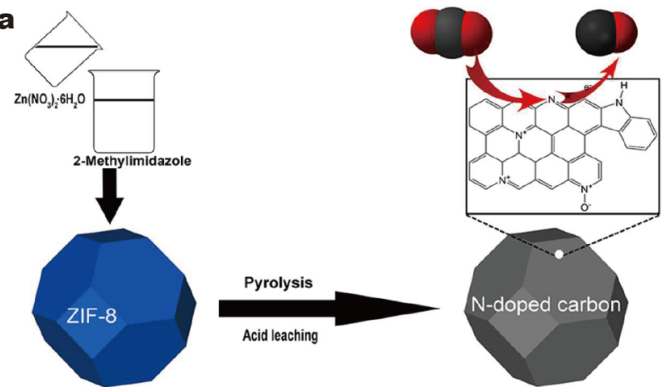

b
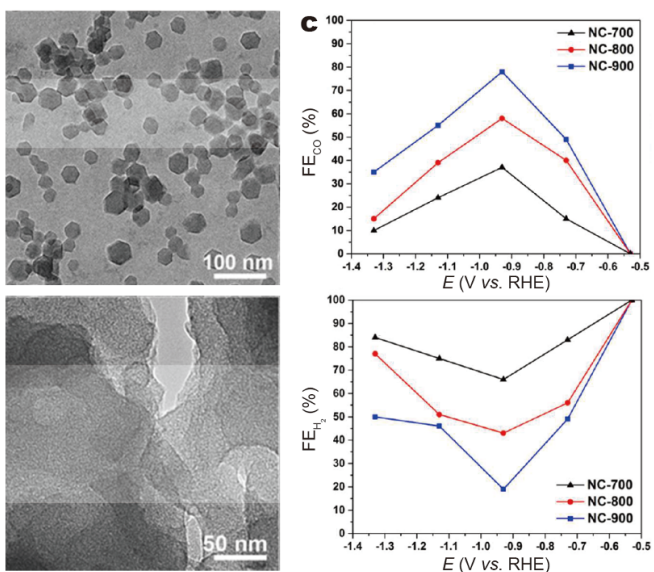
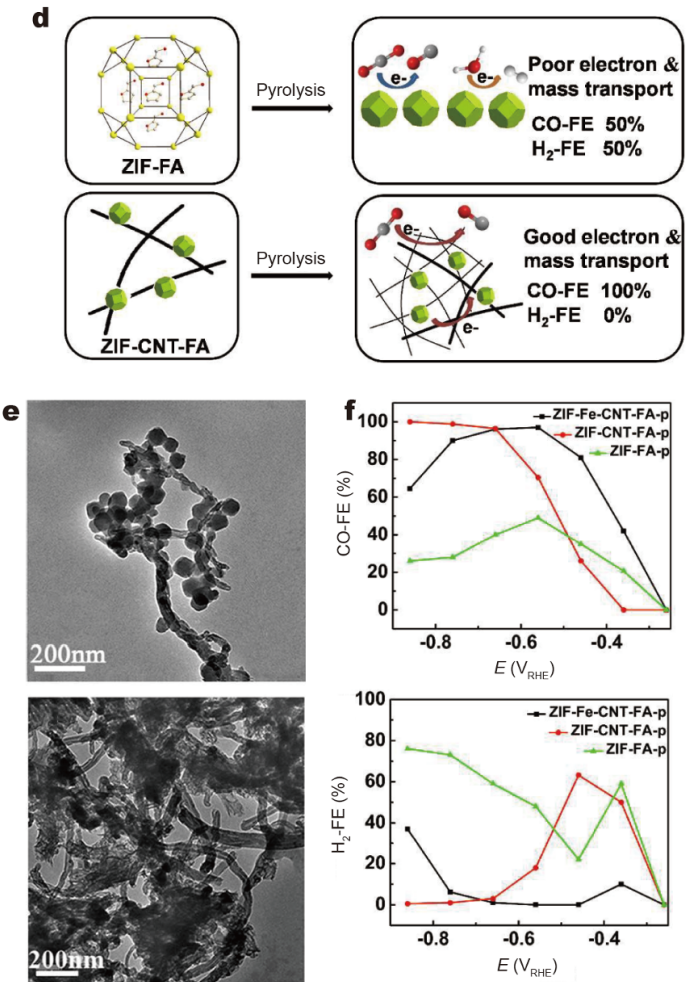

Figure 11 (a) Schematic illustration of the route to synthesize ZIF-8 and N-doped carbon (NC). (b) Transmission electron microscopy (TEM) images of ZIF-8 (upper) and NC-900 (bottom). The agglomeration of dispersed carbon nanoparticles in NC-900 is clear. (c) FEs for CO (upper) and $\mathrm{H}_{2}$ (bottom). The number following NC refers to the pyrolytic temperature. Reprinted with permission from Ref. [189]. Copyright 2018, American Chemical Society. (d) Schematic presentation of enhanced interparticle conductivity and mass transport in MWCNT supported NC. (e) TEM images of ZIF-CNT (upper) and the derived ZIF-CNT-FA-p (bottom). (f) FEs for CO (upper) and $\mathrm{H}_{2}$ (bottom). FA is short for furfuryl alcohol, acting as an additional carbon source. The p refers to pyrolyzed sample. Reprinted with permission from Ref. [196]. Copyright 2018, The Royal Society of Chemistry. 
persed carbon nanoparticles always suffer from agglomerations and inevitable deactivations in long-term applications [192]. Therefore, growing framework materials on robust substrates such as multi-wall carbon nanotubes (MWCNTs) and graphene has been widely utilized to fabricate hybrid materials. Further, thermal decomposition of those composites/mixtures could give more advanced carbon materials [193-195]. For example, Guo et al. [196] prepared a stable $\mathrm{N}$-doped porous carbon by pyrolysis of MWCNTs-supported ZIF-8 and explored its performances for ECR (Fig. 11d, e). The resulting material exhibited higher electrochemically accessible surface areas than that of pyrolyzed ZIF-8 sample without MWCNTs, thus contributing to its high catalytic activity with a current density of $7.7 \mathrm{~mA} \mathrm{~cm}^{-2}$ at $-0.86 \mathrm{~V} v s$. RHE. What is more, with an optimum at $-0.86 \mathrm{~V} v s$. RHE, it displayed a selective $\mathrm{CO}_{2}$-to- $\mathrm{CO}$ reduction in an $\mathrm{FE}$ of nearly $100 \%$ and a steady performance for $10 \mathrm{~h}$ (Fig. 11f). In addition to the high percentage of $\mathrm{N}$ species available for catalytic reactions, the MWCNTs also played pivotal roles in promoting $\mathrm{CO}_{2}$-to-CO reduction for they provided free pathways for electrons and created mesopores, supporting superior electron and mass transports.

\section{MOFs- and COFs-derived metal-based materials}

Porous carbon materials are also attractive for manufacturing carbon-based hybrid catalysts due to their large pore volumes, which allow uniform distribution of the $\mathrm{CO}_{2}$ molecules over the catalysts' surfaces and create massive active sites for ECR $[197,198]$. The carbonization of metal-containing framework materials offers a straightforward way of fabricating porous carbon and metals/metal oxides hybrid materials. Specifically, the porous carbon could be directly casted from organic linkers during the process of high temperature treatment, while the metal nodes could be incorporated into the carbon matrix. In hybrid systems, the activity and selectivity for ECR are affected by the nature of metals, distribution of metal particles and properties of the carbon substrates [199,200].

For example, Zhao and coworkers [201] synthesized oxide-derived $\mathrm{Cu} / \mathrm{carbon}(\mathrm{OD} \mathrm{Cu} / \mathrm{C})$ via a facile pyrolysis of Cu-based MOF (HKUST-1) (Fig. 12a). In the resulting material, $\mathrm{Cu}$ nanoparticles acting as the active sites for ECR were embedded evenly in the porous carbon matrix in an atomic ratio of $4.5 \%$, among which $\mathrm{Cu}_{2} \mathrm{O}$ residues were detected (Fig. 12b-e). When deposited on GC electrode and tested in $\mathrm{CO}_{2}$-saturated aqueous solutions, the resulting material could catalyze $\mathrm{CO}_{2}$ reduction to alcohols (methanol and ethanol) at an onset potential of
$-0.1 \mathrm{~V} v$ s. RHE, corresponding to overpotentials of 120 and $190 \mathrm{mV}$ for methanol and ethanol generations, respectively. Meanwhile, it presented a high selectivity toward alcohol formation, producing methanol with FEs of $13.8 \%-43.2 \%$ and ethanol with FEs of $24 \%-34.8 \%$ at potentials between -0.1 and $-0.7 \mathrm{~V} v$ s. RHE (Fig. 12f, g). Notably, both the FEs and overpotentials for ethanol formation were comparable to the state-of-the art $\mathrm{Cu}$ based catalysts, indicating that such MOF-derived material was potent in $\mathrm{CO}_{2}$-to-alcohol reduction. The improvements in activity and selectivity of the oxide-derived $\mathrm{Cu} /$ carbon could be attributed to the synergistic effects between the highly dispersed copper and the matrix of porous carbon. Firstly, the porous carbon substrate provided a large electroactive area and created high $\mathrm{CO}_{2}$ concentration at the electrode/electrolyte interface, promoting the reaction activity and benefiting the $\mathrm{C}-\mathrm{C}$ coupling [202]. Also, it offers excellent pathways for electron and mass transport, minimizing their related resistances. Besides, the residual $\mathrm{Cu}_{2} \mathrm{O}$ could modify the electronic structure of $\mathrm{Cu}$ and induce a strong binding strength to atomic $\mathrm{O}$, thus reducing the overpotential for ECR and favoring $\mathrm{C}-\mathrm{C}$ coupling. In addition, embedded separately in the carbon matrix, catalytic active $\mathrm{Cu}$ nanoparticles were protected from agglomeration and deactivation, presenting prolonged life for electrocatalysis.

\section{MOFs- and COFs-derived single-atom electrocatalysts}

Commonly, in hybrid materials, metals/metal compounds are prone to aggregate during the thermal treatments, resulting in enlarged nanoparticles and destructed porous structures. What is more, the catalytic sites for ECR are only located on the surface or near surface areas where TPB are formed. Thus, minimizing the sizes of catalytic nanoparticles to expose more reactive sites is important to facilitate the mass/electron transfer at the interface and to achieve high catalytic reactivity. To this end, singleatom electrocatalysts (SAECs) possessing unique electronic and geometric structures have attracted tremendous attentions because they often exhibit unexpected catalytic activity for many important chemical reactions [203-205]. Generally, the rational design and accurate modulation of nanostructured electrocatalysts at the atomic scale are of grand challenges because of the high mobility and diffusivity of subnanometer species $[206,207]$. Actually, framework materials have been demonstrated as excellent precursors or carriers for SAECs because of their unique features allowing sophisticated control over the catalytic motif, support composition and microstructures [208-210]. In 

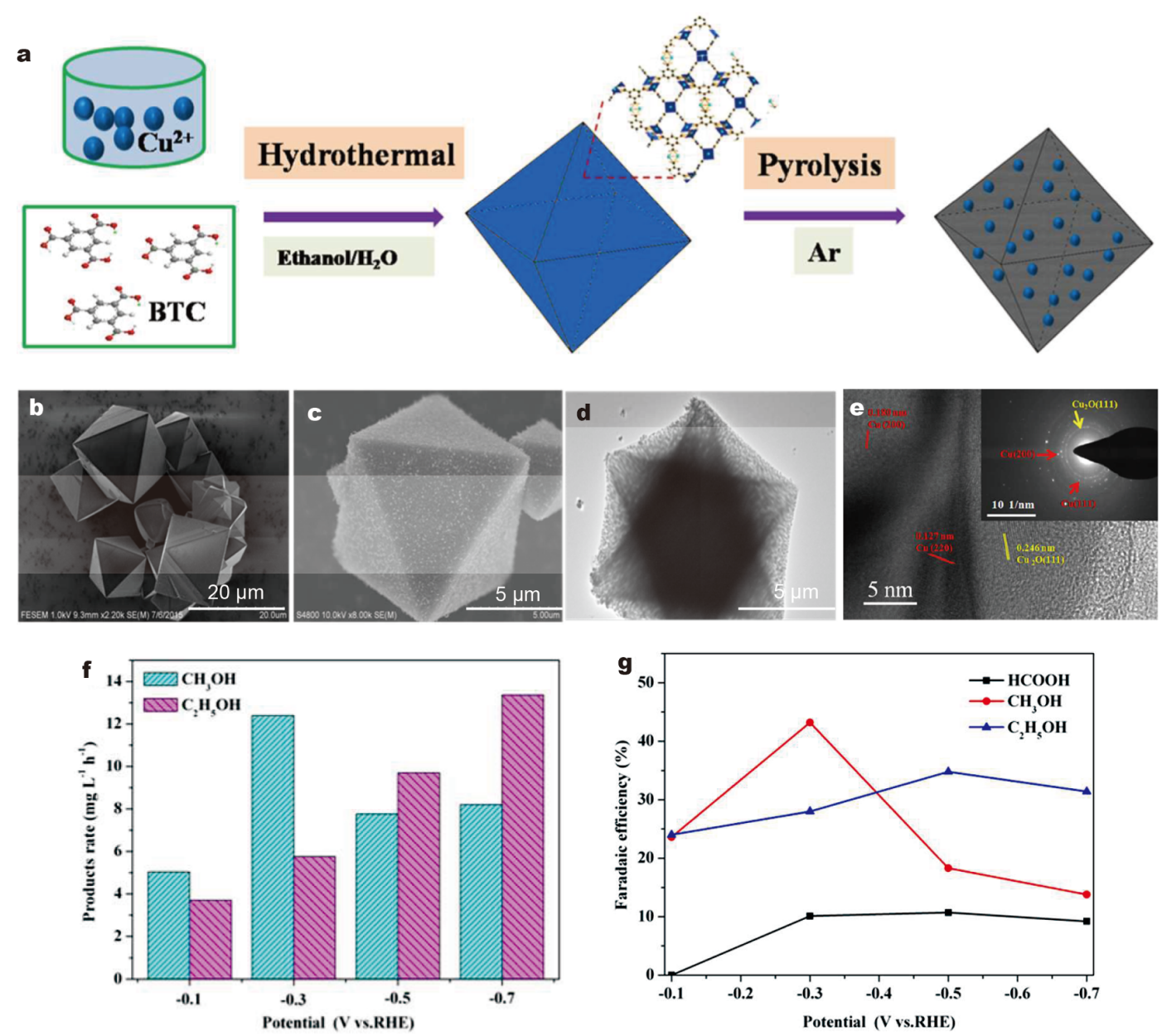

Figure 12 (a) Schematic synthesis process of oxide-derived Cu/carbon catalysts. SEM images of (b) HKUST-1 and (c) OD Cu/C-1000. The number refers to the pyrolytic temperature. (d) TEM image of OD Cu/C-1000. (e) Magnified TEM image and selected area electron diffraction (SAED) patterns of OD Cu/C-1000. (f) Production rates and (g) FEs for ECR products on OD Cu/C-1000 electrode. Reprinted with permission from Ref. [201]. Copyright 2017, American Chemical Society.

this part, we introduce two strategies relating to MOFassisted fabrication of SAECs.

\section{Post-synthetic modification}

Zhao et al. [211] presented a MOF assisted method to prepare single-atom catalyst for ECR, which used post synthetic modifications of pristine ZIF-8 (Fig. 13a). Typically, through a double-solvent approach, $\mathrm{Ni}$ ions were confined within the cavities of ZIF-8. Further pyrolysis induced the transformation of organic ligands into a Ndoped carbon skeleton, where $\mathrm{Ni}$ ions occupied the vacancies caused by $\mathrm{Zn}$ nodes evaporation and were stabilized by $\mathrm{N}$ coordination, generating a hybrid material with $\mathrm{Ni}$ single atoms distributed in $\mathrm{N}$-doped porous carbon (Ni SAs/N-C) (Fig. 13b-g). The homogeneous distribution of $\mathrm{Ni}$ species on the carbon substrate com- bined with dominant $\mathrm{Ni}-\mathrm{N}$ coordination corroborated the atomic dispersion of single $\mathrm{Ni}$ sites (in a ratio of 1.53 at\%) (Fig. 13h). When evaluated in aqueous solutions, it showed a high selectivity towards catalyzing $\mathrm{CO}_{2}$-to- $\mathrm{CO}$ reduction with an $\mathrm{FE}$ of $71.9 \%$ at $-0.9 \mathrm{~V}$ vs. RHE (Fig. 13i). Besides, an ultra-high TOF of $5273 \mathrm{~h}^{-1}$ was achieved at $-1.0 \mathrm{~V} v s$. RHE. Moreover, it exhibited steady electrocatalytic performances for $60 \mathrm{~h}$, during which no obvious decay in FE or current density was detected. Typically, inheriting the high surface area and abundant porosity of ZIF-8, the $\mathrm{Ni}$ SAs/N-C displayed excellent $\mathrm{CO}_{2}$ adsorption capacity and favored electrolyte diffusion/mass transport. Also, the carbon substrate provided an ideal pathway for facile electron transfer, hence lowering the interfacial charge-transfer resistance. Most importantly, the downsizing $\mathrm{Ni}$ to $\mathrm{Ni}$ single-atom catalyst 
dramatically changed its catalytic selectivity (Fig. 13j) $[212,213]$.

Similarly, another single-atom catalyst derived from ZIF-8 was reported by Huan and coworkers [214], in which atomically-dispersed $\mathrm{Fe}$ ions hosted in the carbon matrix and coordinated with $\mathrm{N}$ and/or $\mathrm{C}$ atoms (Fig. 14a), forming a typical Fe-N-C material. In an optimized sample, the material containing $\mathrm{Fe}-\mathrm{N}_{4}$ moieties in a ratio of $100 \%$ displayed superior activity and selectivity in catalyzing $\mathrm{CO}_{2}$-to-CO reduction in aqueous solutions (Fig. 14b), in terms of low onset potential $(-0.3 \mathrm{~V} v s$. RHE, overpotential of $190 \mathrm{mV}$ ), high FE (up to 91\%), and sustaining long-term electrolysis (stable current density of $6 \mathrm{~mA} \mathrm{~cm}^{-2}$ for $6 \mathrm{~h}$ ). Notably, owing to the flexibility of the MOF-assisted strategy, other Fe-containing moieties (e.g., $\mathrm{FeC}_{3}$ and $\mathrm{Fe}$ crystalline nanoparticles) could be introduced facilely (Fig. 14c), therefore enabling the identification of their roles in hybrid systems. Specifically, the presence of $\mathrm{Fe}$ carbide and $\mathrm{Fe}$ crystalline nanoparticles resulted in high $\mathrm{FE}$ for $\mathrm{H}_{2}$ evolution, indicating that the isolated $\mathrm{Fe}-\mathrm{N}_{4}$ sites were critical for the selective $\mathrm{CO}_{2}$ reduction into CO (Fig. 14d, e) [215]. Although most of $\mathrm{Zn}$ species in ZIFs were removed during the pyrolysis and acid-leaching processes, there were still residual $\mathrm{Zn}$ ions anchored into the carbon matrix with $\mathrm{N}$ sites, which inevitably interfered with the exposure of $\mathrm{Fe}-\mathrm{N}_{4}$ sites and restricted their catalytic activity. To tackle this issue, Yang and coworkers [216] explored an additional ammonia treatment process to facilitate the sublimation of residual $\mathrm{Zn}$ species and unstable carbon moieties, thus producing a hierarchical porous structure with larger specific surface area and more exposed $\mathrm{Fe}-\mathrm{N}_{4}$ sites. Consequently, an optimal catalyst synthesized from such method showed much improved activity with a $\mathrm{CO}$ current density of $17.8 \mathrm{~mA} \mathrm{~cm}^{-2}$, outperforming the material prepared without a second ammonia reduction. Besides, the ammonia treatment introduced more mesopores into the Fe$\mathrm{N}-\mathrm{C}$ catalyst which further promoted the mass transfer during the ECR and favored the high-rate electrocatalysis.

\section{In situ substitution}

In addition to filtrating ions into the pores, ionic exchanges could happen in the frameworks by substituting metal centers with other metal ions, thus providing an alternative strategy to produce SAECs (Fig. 15a) $[217,218]$. For instance, Song and coworkers [219] prepared a Co-based SAEC supported by nitrogen-doped 3D hollow carbon structure (Co-HNC) from a $\mathrm{Zn} / \mathrm{Co}$ bimetallic ZIF grown on ZnO nanospheres (ZnO@ZIF). The presence of $\mathrm{Zn}$ nodes in the frameworks could dilute the concentration of $\mathrm{Co}$, which in combination with the excess $\mathrm{Zn}$ evaporation from $\mathrm{ZnO}$ template effectively prevented aggregation of Co single atoms during the pyrolysis process. Moreover, the in situ reduction and evaporation of $\mathrm{ZnO}$ cores created hollow interiors and sponge-like carbon shells (Fig. 15b-l), forming hierarchical pore systems with more mesopores and allowing maximal exposure of active sites for triple-phase electrocatalytic reactions. With Co single atoms uniformly coordinated in the form of Co- $\mathrm{C}_{2} \mathrm{~N}_{2}$ moieties (Fig. $15 \mathrm{~m}$ ), such material worked as a bifunctional catalyst for ECR and HER in aqueous solutions and generated $\mathrm{CO}$ and $\mathrm{H}_{2}$ in a ratio approximating $1 / 2$ within a wide potential window (nearly $100 \% \mathrm{FE}$ in total) (Fig. 15n), thus farming it as a promising electrocatalyst for syngas production.

\section{Short summary}

Generally, those MOF-derived materials to some extent inherited advantageous features (e.g., large surface areas and porous structures) from pristine MOFs and demonstrated competitive performances in catalyzing ECR. Compared with pristine MOFs, whether in those carbon materials or metal compound/carbon hybrids, the carbon matrixes obtained from the organic ligands provided fast electron transfer pathways, thus inducing improved electronic conductivity. Also, those carbon substrates prevented the aggregation of metal/metallic compounds nanoparticles or the collapse of integrated architectures in long-term operations. Besides, heteroatom-doping could be realized easily, thus offering opportunities to tune the electron distribution and to create favorable microenvironment for $\mathrm{CO}_{2}$ reduction.

Albeit some physiochemical features of MOF-derived materials can be controlled by tuning the pyrolysis processes, the limited knowledge of the transformation mechanism restricts their rational design. Therefore, in situ experimental and characterization techniques should be introduced to further understand those systems.

\section{SUMMARY AND PERSPECTIVES}

Among tremendous strategies aiming to mitigate the negative effects of $\mathrm{CO}_{2}$ accumulation in the atmosphere, reducing $\mathrm{CO}_{2}$ into carbonaceous fuels electrochemically seems to hold great promise due to the increasing amount of electricity generated from intermittent renewable energies. For practical applications, since the ECR is a complex process which produces various kinds of products and always suffers from sluggish kinetics, exploring robust electrocatalysts being able to reduce $\mathrm{CO}_{2}$ selectively and actively is highly desired. In the past few years, 

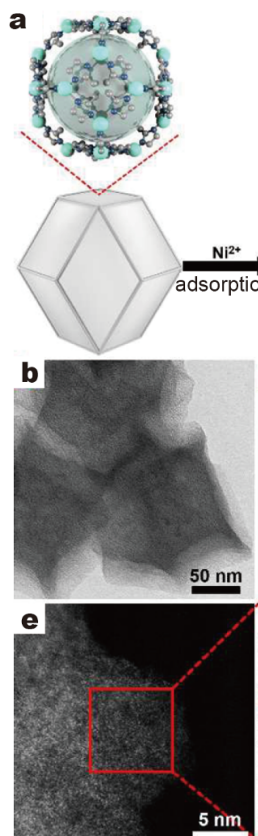
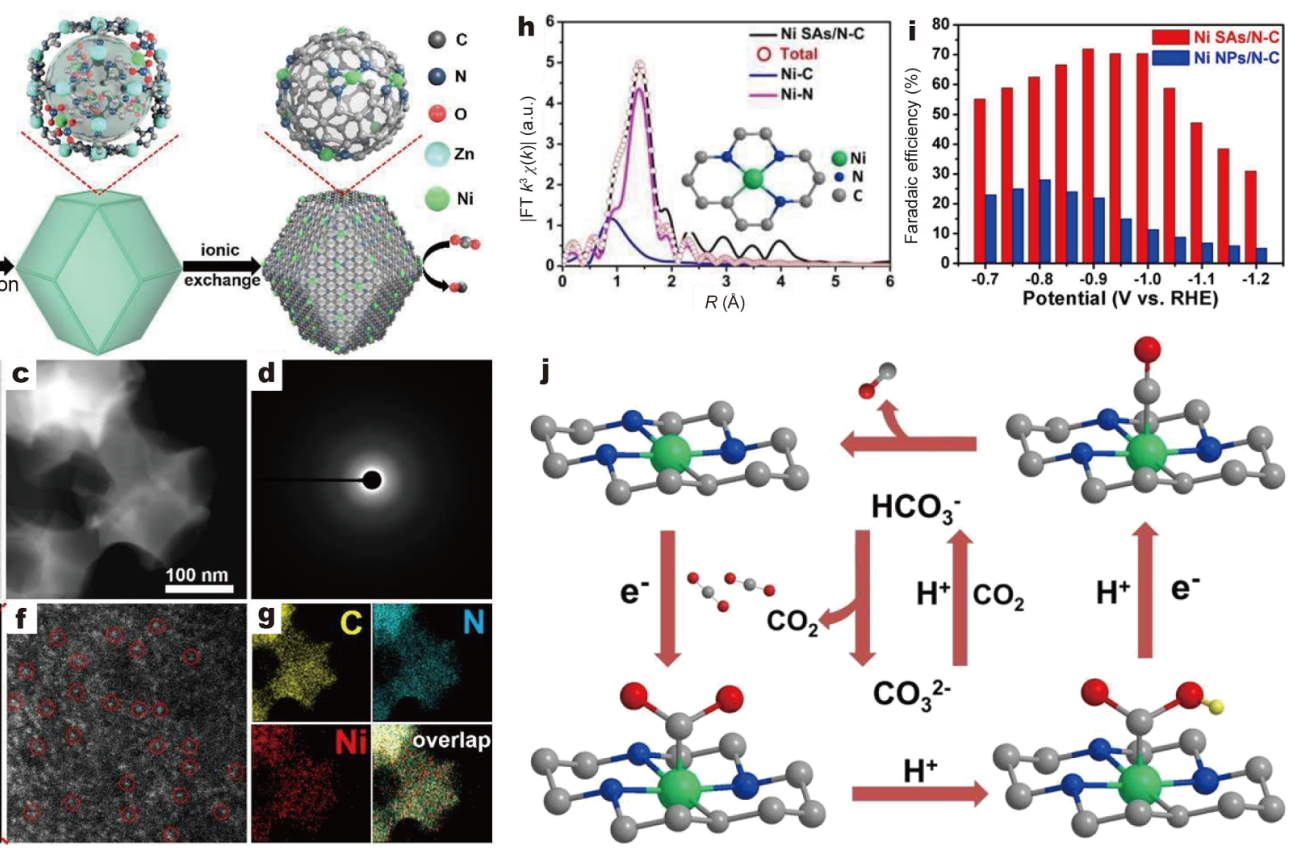

$\mathrm{HCO}_{3}{ }^{-}$
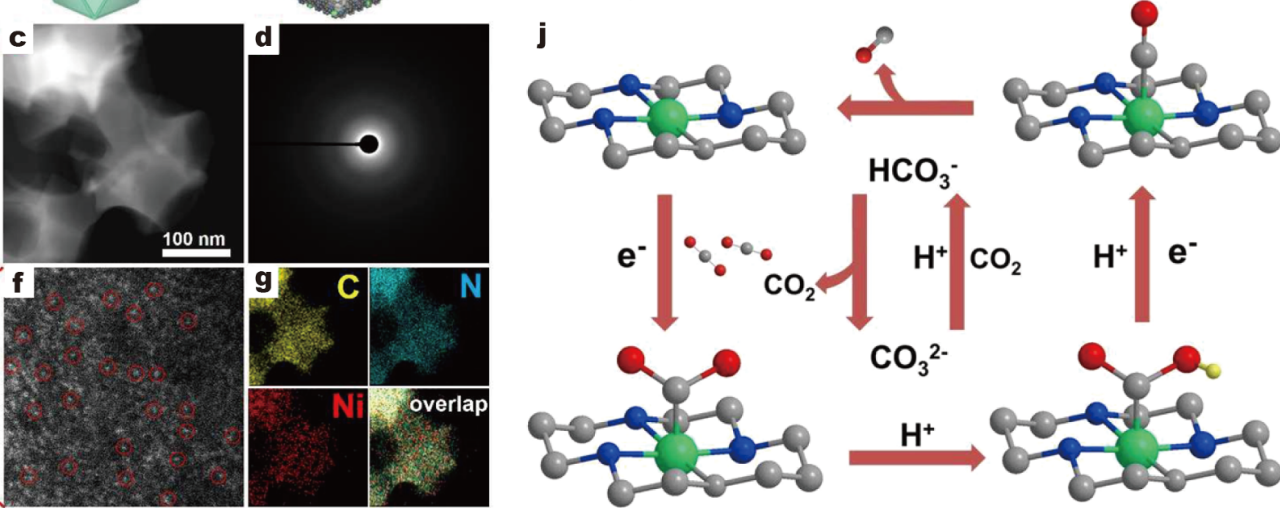

Figure 13 (a) Scheme of the formation of Ni SAs/N-C. (b) TEM and (c) high-angle annular dark-field scanning TEM (HAADF-STEM) images of Ni SAs/N-C. (d) Corresponding SAED pattern of an individual rhombic dodecahedron. (e, f) Magnified HAADF-STEM images of Ni SAs/N-C. The Ni single atoms are marked with red circles. (g) Corresponding EDS mapping images revealing the homogeneous distribution of $\mathrm{Ni}$ and $\mathrm{N}$ on the carbon support. (h) X-ray absorption near-edge structure (XANES) spectra and corresponding fitting curves of Ni SAs/N-C. Inset is the proposed Ni-N moiety. (i) FEs for $\mathrm{CO}$ on Ni SAs/N-C and Ni NPs/N-C. NP refers to nanoparticle, formed by the accumulation of single atoms and crystal growth when excess Ni ions were adsorbed. (j) Proposed reaction paths for $\mathrm{CO}_{2}$ electroreduction on Ni SAs/N-C. Reprinted with permission from Ref. [211]. Copyright 2017, American Chemical Society.

MOFs- and COFs-based materials have demonstrated great potentials in catalyzing ECR due to their high specific surface areas and porous structures that provide more exposed active sites and facile mass transport pathways. Also, the emerging reticular chemistry makes tuning their electronic and geometric features on the atomic/molecular levels possible, thus satisfying their modifications to favor a specific product in ECR [220222].

Albeit advances have been achieved, employing framework material-based catalysts for ECR is still in its infancy and a systematic investigation is needed to figure out how those intrinsic characteristics of MOFs/COFs affect their reactivity in catalyzing ECR. Whilst, more efforts should be dedicated with the following concerns that prohibit framework-based materials from practical applications. Firstly, the majority of pristine MOFs/COFs usually display poor electronic conductivity, which is the bottleneck for achieving high efficiency in ECR. Therefore, developing conductive MOFs/COFs and hybridizing them with conductive materials (e.g., carbon-based materials) will create new opportunities to design highly efficient electrocatalysts. Secondly, the poor stability of
MOFs/COFs in water caused by the leaching of metal species or organic components usually incurs inevitable performance decays in long-term electrolysis. In detail, the coordinatively unsaturated metal centers in MOFs/ COFs are prone to react with water molecules and dissolve from the frameworks. Thus, modifying MOFs/COFs with hydrophobic groups while preserving their facile mass transports is highly desired [223]. Besides, inspired by the function of solid electrolyte interfaces (SEI) fully investigated in energy storage systems [224,225], encapsulating MOFs/COFs into protective matrixes should be conceivable to block those active centers from chemical attack and to counter their deterioration. Except the electrolyte-induced degradation, the dispersed metal centers undergoing redox reactions might agglomerate and leach from the frameworks when they are reduced to the zero valance states, similar to the common deactivation mode in molecular complexes. Therefore, constructing MOFs/COFs with conjugated electron structures is of great importance to stabilize the metal centers even in their atomic states. Whilst, in some molecular catalysts, the redox reactions occur on the organic ligands or on both the metal centers and organic ligands, 


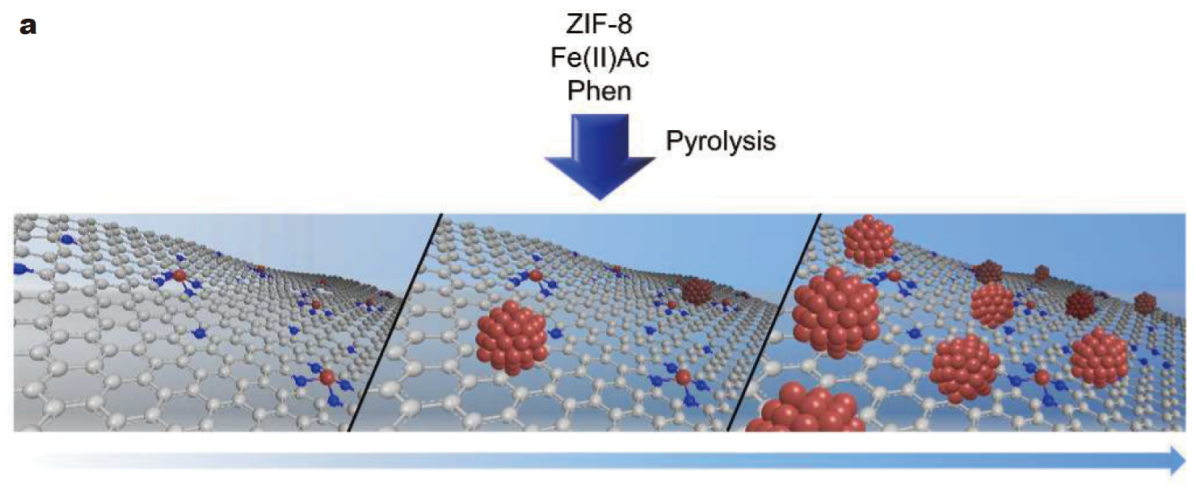

$\mathrm{Fe}_{0.5} \mathrm{~d}$
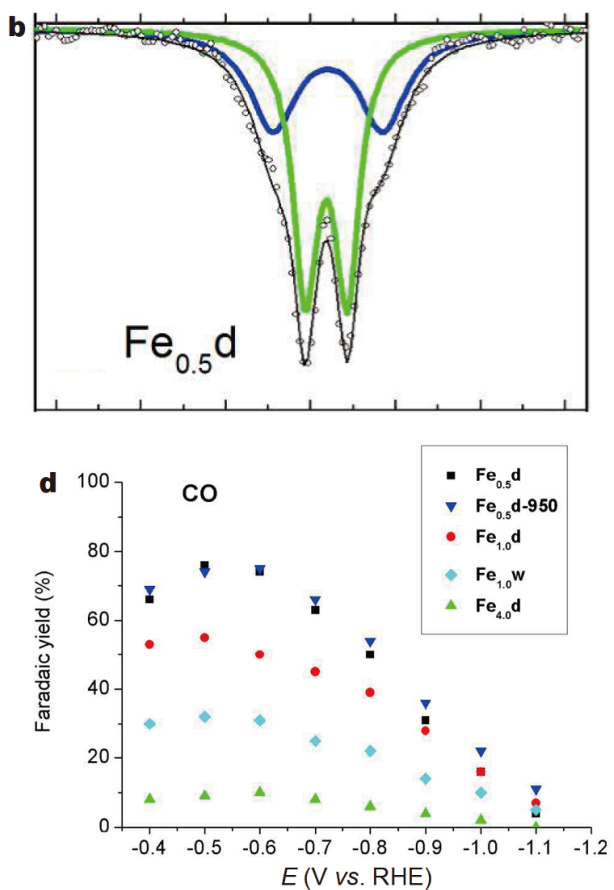
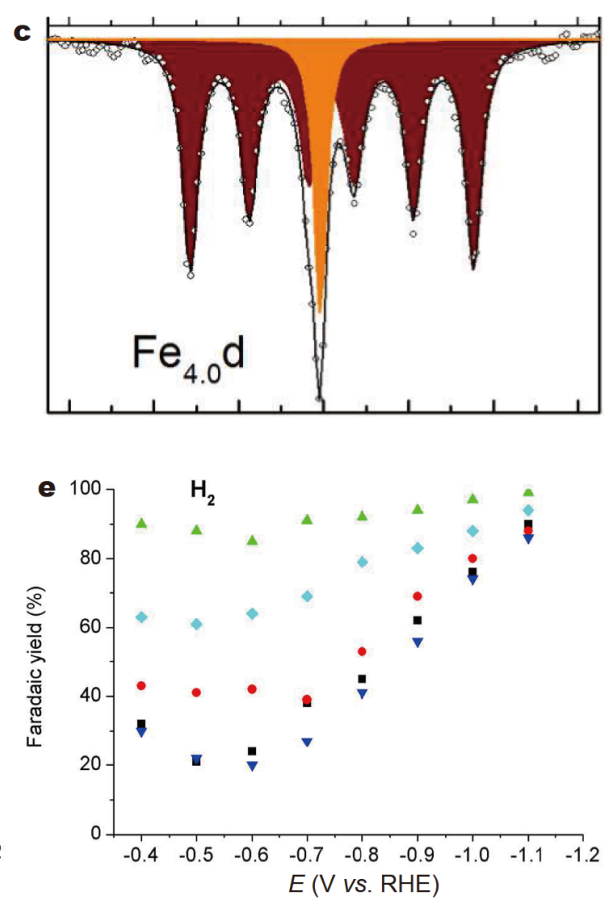

Figure 14 (a) Schematic representation of the various Fe-N-C materials obtained upon pyrolysis: the amount of Fe nanoparticles increases with increased Fe loading. Fe atoms are represented in red, $\mathrm{C}$ atoms in grey and $\mathrm{N}$ atoms in blue. The graphitic shell typically surrounding Fe nanoparticles after pyrolysis is not represented for clarity. (b, c) ${ }^{57} \mathrm{Fe}$ Mössbauer absorption spectra of Fe-N-C materials, as labelled on the figures. FEs for (d) CO and (e) $\mathrm{H}_{2}$ formation of those materials. Reprinted with permission form Ref. [214]. Copyright 2017, American Chemical Society.

thus providing valuable clues on designing various MOFs/COFs with stable redox centers [226]. The organic framework derivatives, with partially inherited characteristics of pristine MOFs/COFs, have addressed some issues associated with their parent materials, namely electronic conductivity and stability. However, precise control on the electronic and morphologic parameters of these materials is absent because of the limited knowledge of the transformation process, inhibiting their rational designs at this stage. Thus, uncovering the possible migration and disordering of compositional components may elucidate the transformative trends and help fabri- cate more advanced organic framework-derived electrocatalysts.

Given that we have learned more about the ECR mechanisms according to recent progresses in both experimental and theoretical trials, it should be possible to control which pathway dominates at the branching point via artificially tuning the electric fields of MOFs or COFs. For example, a strong binding energy between * $\mathrm{CO}$ and the catalyst promotes its further reduction and a large amount of *CO coverage favors $\mathrm{C}-\mathrm{C}$ dimerization. In common, knowing the intermediates and producing intermediate-rich local environments are pivotal pre- 

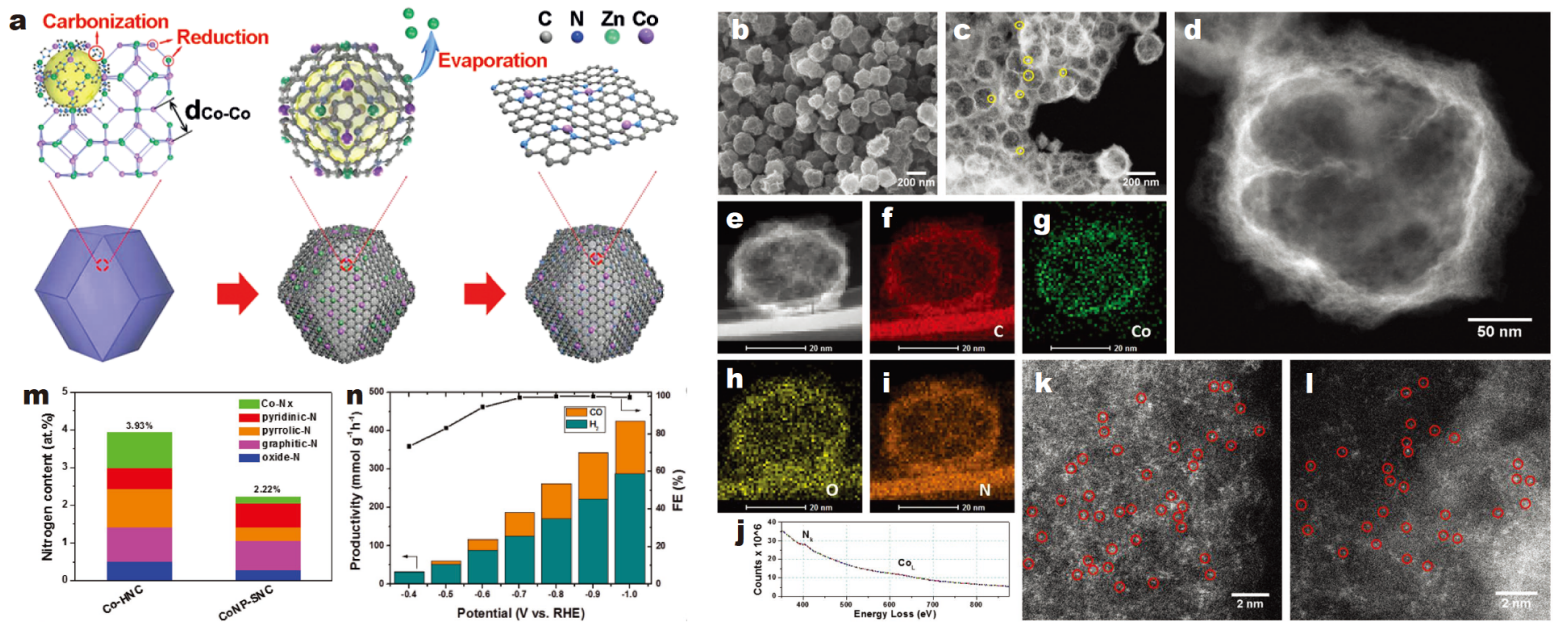

Figure 15 (a) Schematic formation of Co single sites in the Zn/Co bimetallic ZIF. Reprinted with permission from Ref. [207]. Copyright 2016, WileyVCH. (b) SEM and (c, d) STEM images of Co-HNC. (e-i) Corresponding electron energy loss (EEL) mapping images indicating homogeneous distribution of $\mathrm{N}, \mathrm{O}$, and Co throughout the hollow carbon structure. (j) EEL spectra of Co-HNC. (k, l) HAADF-STEM images of Co-HNC at different areas. Part of Co single atoms are marked with red circles. $(\mathrm{m})$ Atomic contents of $\mathrm{N}$ species in Co-HNC and Co NP-SNC (solid nitrogendoped carbon embedded with Co nanoparticle). Inset is the proposed $\mathrm{Co}_{-} \mathrm{C}_{2} \mathrm{~N}_{2}$ moiety. (n) Productivity (left $Y$-axis) and FE (right $Y$-axis) of CoHNC. Reprinted with permission from Ref. [219]. Copyright 2018, Wiley-VCH.

requisites to increase the catalytic selectivity towards valuable hydrocarbons, and they should be taken into consideration in designing electrocatalysts.

In summary, as an effective electrocatalyst for ECR should possess optimal electronic and geometric characteristics favoring rapid and stable generation of a desired product, framework-based materials being able to be tuned precisely provide ideal platforms to design advanced electrocatalysts and to explore how those features affect the catalytic properties. Looking forward, in addition to experimentally achieving predesigned compositions, components, and functions via atomically precise integration of building blocks and metal centers, conducting theoretical calculations is urgently required to gain deep insight into catalytic mechanisms and to generate design rules for new catalysts [227]. Additionally, on the other side of the electrode-electrolyte interfaces, the liquid media also play pivotal roles in determining the binding affinities of critical reaction intermediates, thereby influencing the selectivity and efficiency of ECR $[228,229]$. From this perspective, engineering catalytically promoting electrolytes compatible with advanced framework material-based electrodes will alleviate many barriers of ECR and engender reliable systems for practical applications. Granted, deliberate design for frameworkbased materials might pave the way for fabricating excellent ECR catalysts in laboratories, but exploring largescale synthetic methods with high yields and affordable cost is required for industrial applications. Clearly, in the coming years, the increasing researches geared toward fabricating MOFs- and COFs-based materials will create unparalleled opportunities to fabricate advanced electrocatalysts for ECR.

Received 10 January 2020; accepted 17 March 2020; published online 28 April 2020

1 Chu S, Majumdar A. Opportunities and challenges for a sustainable energy future. Nature, 2012, 488: 294-303

2 Mac Dowell N, Fennell PS, Shah N, et al. The role of $\mathrm{CO}_{2}$ capture and utilization in mitigating climate change. Nat Clim Change, 2017, 7: 243-249

3 Leitner W, Quadrelli EA, Schlögl R. Harvesting renewable energy with chemistry. Green Chem, 2017, 19: 2307-2308

4 Drechsler M, Egerer J, Lange M, et al. Efficient and equitable spatial allocation of renewable power plants at the country scale. Nat Energy, 2017, 2: 17124

5 Siria A, Bocquet ML, Bocquet L. New avenues for the large-scale harvesting of blue energy. Nat Rev Chem, 2017, 1: 0091

6 British Petroleum (BP). Statistical Review of World Energy 2018. BP, 2018

7 Peters M, Köhler B, Kuckshinrichs W, et al. Chemical technologies for exploiting and recycling carbon dioxide into the value chain. ChemSusChem, 2011, 4: 1216-1240

8 Zhang N, Long R, Gao C, et al. Recent progress on advanced design for photoelectrochemical reduction of $\mathrm{CO}_{2}$ to fuels. Sci China Mater, 2018, 61: 771-805

9 Rau GH, Willauer HD, Ren ZJ. The global potential for converting renewable electricity to negative- $\mathrm{CO}_{2}$-emissions hydrogen. Nat Clim Change, 2018, 8: 621-625

10 Bickle MJ. Geological carbon storage. Nat Geosci, 2009, 2: 815818

11 Sanna A, Uibu M, Caramanna G, et al. A review of mineral 
carbonation technologies to sequester $\mathrm{CO}_{2}$. Chem Soc Rev, 2014, 43: 8049-8080

12 Albo J, Alvarez-Guerra M, Castaño P, et al. Towards the electrochemical conversion of carbon dioxide into methanol. Green Chem, 2015, 17: 2304-2324

13 Aresta M, Dibenedetto A, Angelini A. Catalysis for the valorization of exhaust carbon: From $\mathrm{CO}_{2}$ to chemicals, materials, and fuels. Technological use of $\mathrm{CO}_{2}$. Chem Rev, 2014, 114: 1709-1742

14 Apaydin DH, Schlager S, Portenkirchner E, et al. Organic, organometallic and bioorganic catalysts for electrochemical reduction of $\mathrm{CO}_{2}$. ChemPhysChem, 2017, 18: 3094-3116

15 Zhang $\mathrm{H}$, Zhang $\mathrm{Y}, \mathrm{Li} \mathrm{Y}$, et al. Cu nanowire-catalyzed electrochemical reduction of $\mathrm{CO}$ or $\mathrm{CO}_{2}$. Nanoscale, 2019, 11: 1207512079

16 Li F, MacFarlane DR, Zhang J. Recent advances in the nanoengineering of electrocatalysts for $\mathrm{CO}_{2}$ reduction. Nanoscale, 2018, 10: 6235-6260

17 Zhang $\mathrm{W}, \mathrm{Hu} \mathrm{Y}, \mathrm{Ma} \mathrm{L}$, et al. Progress and perspective of electrocatalytic $\mathrm{CO}_{2}$ reduction for renewable carbonaceous fuels and chemicals. Adv Sci, 2018, 5: 1700275

$18 \mathrm{Li} \mathrm{J}$, Chen G, Zhu Y, et al. Efficient electrocatalytic $\mathrm{CO}_{2}$ reduction on a three-phase interface. Nat Catal, 2018, 1: 592-600

19 Kas R, Hummadi KK, Kortlever R, et al. Three-dimensional porous hollow fibre copper electrodes for efficient and high-rate electrochemical carbon dioxide reduction. Nat Commun, 2016, 7: 10748

20 Costentin C, Robert M, Savéant JM. Catalysis of the electrochemical reduction of carbon dioxide. Chem Soc Rev, 2013, 42: 2423-2436

21 Peterson AA, Nørskov JK. Activity descriptors for $\mathrm{CO}_{2}$ electroreduction to methane on transition-metal catalysts. J Phys Chem Lett, 2012, 3: 251-258

22 Klingan K, Kottakkat T, Jovanov ZP, et al. Reactivity determinants in electrodeposited $\mathrm{Cu}$ foams for electrochemical $\mathrm{CO}_{2}$ reduction. ChemSusChem, 2018, 11: 3449-3459

23 Miner EM, Gul S, Ricke ND, et al. Mechanistic evidence for ligand-centered electrocatalytic oxygen reduction with the conductive MOF $\mathrm{Ni}_{3}$ (hexaiminotriphenylene) ${ }_{2}$. ACS Catal, 2017, 7: 7726-7731

24 Wang B, Côté AP, Furukawa H, et al. Colossal cages in zeolitic imidazolate frameworks as selective carbon dioxide reservoirs. Nature, 2008, 453: 207-211

25 Jiang K, Zhang L, Xia T, et al. A water-stable fcu-MOF material with exposed amino groups for the multi-functional separation of small molecules. Sci China Mater, 2019, 62: 1315-1322

26 Jiang J, Zhao Y, Yaghi OM. Covalent chemistry beyond molecules. J Am Chem Soc, 2016, 138: 3255-3265

27 Diercks C, Kalmutzki M, Yaghi O. Covalent organic frameworks -Organic chemistry beyond the molecule. Molecules, 2017, 22: 1575-1581

28 Cao L, Tao P, Li M, et al. Synergistic effects of C/a-MoC and Ag for efficient oxygen reduction reaction. J Phys Chem Lett, 2018, 9: 779-784

$29 \mathrm{Gu} \mathrm{S}, \mathrm{Wu}$ S, Cao L, et al. Tunable redox chemistry and stability of radical intermediates in $2 \mathrm{D}$ covalent organic frameworks for high performance sodium ion batteries. J Am Chem Soc, 2019, 141: 9623-9628

30 Liu J, Zhu D, Guo C, et al. Design strategies toward advanced MOF-derived electrocatalysts for energy-conversion reactions. Adv Energy Mater, 2017, 7: 1700518
31 Cao L, Lv F, Liu $\mathrm{Y}$, et al. A high performance $\mathrm{O}_{2}$ selective membrane based on CAU-1- $\mathrm{NH}_{2} @$ polydopamine and the PMMA polymer for Li-air batteries. Chem Commun, 2015, 51: 4364-4367

32 Nath I, Chakraborty J, Verpoort F. Metal organic frameworks mimicking natural enzymes: A structural and functional analogy. Chem Soc Rev, 2016, 45: 4127-4170

33 Morozan A, Jaouen F. Metal organic frameworks for electrochemical applications. Energy Environ Sci, 2012, 5: 9269-9290

34 Wu S, Zhu Y, Huo Y, et al. Bimetallic organic frameworks derived $\mathrm{CuNi} /$ carbon nanocomposites as efficient electrocatalysts for oxygen reduction reaction. Sci China Mater, 2017, 60: 654-663

35 Hendon $\mathrm{CH}$, Rieth AJ, Korzyński MD, et al. Grand challenges and future opportunities for metal-organic frameworks. ACS Cent Sci, 2017, 3: 554-563

36 Yaghi OM. Reticular chemistry-construction, properties, and precision reactions of frameworks. J Am Chem Soc, 2016, 138: 15507-15509

37 Park SS, Rieth AJ, Hendon $\mathrm{CH}$, et al. Selective vapor pressure dependent proton transport in a metal-organic framework with two distinct hydrophilic pores. J Am Chem Soc, 2018, 140: 20162019

38 Xie LS, Sun L, Wan R, et al. Tunable mixed-valence doping toward record electrical conductivity in a three-dimensional metalorganic framework. J Am Chem Soc, 2018, 140: 7411-7414

39 Hod I, Farha OK, Hupp JT. Powered by porphyrin packing. Nat Mater, 2015, 14: 1192-1193

40 Hansen HA, Varley JB, Peterson AA, et al. Understanding trends in the electrocatalytic activity of metals and enzymes for $\mathrm{CO}_{2}$ reduction to CO. J Phys Chem Lett, 2013, 4: 388-392

41 Seifitokaldani A, Gabardo CM, Burdyny T, et al. Hydroniuminduced switching between $\mathrm{CO}_{2}$ electroreduction pathways. J Am Chem Soc, 2018, 140: 3833-3837

42 Feaster JT, Shi C, Cave ER, et al. Understanding selectivity for the electrochemical reduction of carbon dioxide to formic acid and carbon monoxide on metal electrodes. ACS Catal, 2017, 7: 48224827

43 Chen J, Wang Z, Lee $\mathrm{H}$, et al. Efficient electroreduction of $\mathrm{CO}_{2}$ to $\mathrm{CO}$ by Ag-decorated S-doped $\mathrm{g}-\mathrm{C}_{3} \mathrm{~N}_{4} / \mathrm{CNT}$ nanocomposites at industrial scale current density. Mater Today Phys, 2020, 12: 100176

44 Cheng T, Xiao H, Goddard Iii WA. Full atomistic reaction mechanism with kinetics for $\mathrm{CO}$ reduction on $\mathrm{Cu}(100)$ from $a b$ initio molecular dynamics free-energy calculations at $298 \mathrm{~K}$. Proc Natl Acad Sci USA, 2017, 114: 1795-1800

45 Montoya JH, Shi C, Chan $\mathrm{K}$, et al. Theoretical insights into a CO dimerization mechanism in $\mathrm{CO}_{2}$ electroreduction. J Phys Chem Lett, 2015, 6: 2032-2037

46 Schouten KJP, Kwon Y, van der Ham CJM, et al. A new mechanism for the selectivity to $C_{1}$ and $C_{2}$ species in the electrochemical reduction of carbon dioxide on copper electrodes. Chem Sci, 2012, 2: 1902-1909

47 Luo M, Wang Z, Li YC, et al. Hydroxide promotes carbon dioxide electroreduction to ethanol on copper via tuning of adsorbed hydrogen. Nat Commun, 2019, 10: 5814

48 Li YC, Wang Z, Yuan T, et al. Binding site diversity promotes $\mathrm{CO}_{2}$ electroreduction to ethanol. J Am Chem Soc, 2019, 141: 8584-8591

49 Goodpaster JD, Bell AT, Head-Gordon M. Identification of possible pathways for $\mathrm{C}-\mathrm{C}$ bond formation during electrochemical 
reduction of $\mathrm{CO}_{2}$ : New theoretical insights from an improved electrochemical model. J Phys Chem Lett, 2016, 7: 1471-1477

50 Li J, Wang Z, McCallum C, et al. Constraining CO coverage on copper promotes high-efficiency ethylene electroproduction. Nat Catal, 2019, 2: 1124-1131

51 Wang X, Xu A, Li F, et al. Efficient methane electrosynthesis enabled by tuning local $\mathrm{CO}_{2}$ availability. J Am Chem Soc, 2020, 142: 3525-3531

52 Wang Y, Wang Z, Dinh CT, et al. Catalyst synthesis under $\mathrm{CO}_{2}$ electroreduction favours faceting and promotes renewable fuels electrosynthesis. Nat Catal, 2020, 3: 98-106

53 Liu M, Liu M, Wang X, et al. Quantum-dot-derived catalysts for $\mathrm{CO}_{2}$ reduction reaction. Joule, 2019, 3: 1703-1718

54 Ross MB, De Luna P, Li Y, et al. Designing materials for electrochemical carbon dioxide recycling. Nat Catal, 2019, 2: 648-658

55 Li F, Li YC, Wang Z, et al. Cooperative $\mathrm{CO}_{2}$-to-ethanol conversion via enriched intermediates at molecule-metal catalyst interfaces. Nat Catal, 2020, 3: 75-82

56 Li F, Thevenon A, Rosas-Hernández A, et al. Molecular tuning of $\mathrm{CO}_{2}$-to-ethylene conversion. Nature, 2020, 577: 509-513

57 Zhao G, Huang X, Wang X, et al. Progress in catalyst exploration for heterogeneous $\mathrm{CO}_{2}$ reduction and utilization: A critical review. J Mater Chem A, 2017, 5: 21625-21649

58 Kuhl KP, Hatsukade T, Cave ER, et al. Electrocatalytic conversion of carbon dioxide to methane and methanol on transition metal surfaces. J Am Chem Soc, 2014, 136: 14107-14113

59 Gattrell M, Gupta N, Co A. A review of the aqueous electrochemical reduction of $\mathrm{CO}_{2}$ to hydrocarbons at copper. J Electroanal Chem, 2006, 594: 1-19

60 Peterson AA, Abild-Pedersen F, Studt F, et al. How copper catalyzes the electroreduction of carbon dioxide into hydrocarbon fuels. Energy Environ Sci, 2010, 3: 1311-1315

$61 \mathrm{Lu} \mathrm{Q}$, Jiao F. Electrochemical $\mathrm{CO}_{2}$ reduction: Electrocatalyst, reaction mechanism, and process engineering. Nano Energy, 2016, 29: $439-456$

62 Ma M, Trześniewski BJ, Xie J, et al. Selective and efficient reduction of carbon dioxide to carbon monoxide on oxide-derived nanostructured silver electrocatalysts. Angew Chem Int Ed, 2016, 55: 9748-9752

63 Durand WJ, Peterson AA, Studt F, et al. Structure effects on the energetics of the electrochemical reduction of $\mathrm{CO}_{2}$ by copper surfaces. Surf Sci, 2011, 605: 1354-1359

64 Chen Y, Li CW, Kanan MW. Aqueous $\mathrm{CO}_{2}$ reduction at very low overpotential on oxide-derived $\mathrm{Au}$ nanoparticles. J Am Chem Soc, 2012, 134: 19969-19972

65 Zhang Y, Zhang X, Bond AM, et al. Identification of a new substrate effect that enhances the electrocatalytic activity of dendritic tin in $\mathrm{CO}_{2}$ reduction. Phys Chem Chem Phys, 2018, 20: 5936-5941

$66 \mathrm{Gu}$ J, Héroguel F, Luterbacher J, et al. Densely packed, ultra small $\mathrm{SnO}$ nanoparticles for enhanced activity and selectivity in electrochemical $\mathrm{CO}_{2}$ reduction. Angew Chem Int Ed, 2018, 57: 29432947

67 Huo S, Weng Z, Wu Z, et al. Coupled metal/oxide catalysts with tunable product selectivity for electrocatalytic $\mathrm{CO}_{2}$ reduction. ACS Appl Mater Interfaces, 2017, 9: 28519-28526

68 Hall AS, Yoon Y, Wuttig A, et al. Mesostructure-induced selectivity in $\mathrm{CO}_{2}$ reduction catalysis. J Am Chem Soc, 2015, 137: 14834-14837

69 Yoon Y, Hall AS, Surendranath Y. Tuning of silver catalyst me- sostructure promotes selective carbon dioxide conversion into fuels. Angew Chem Int Ed, 2016, 55: 15282-15286

70 Larrazábal GO, Shinagawa T, Martín AJ, et al. Microfabricated electrodes unravel the role of interfaces in multicomponent copper-based $\mathrm{CO}_{2}$ reduction catalysts. Nat Commun, 2018, 9: 1477

71 Voiry D, Shin HS, Loh KP, et al. Low-dimensional catalysts for hydrogen evolution and $\mathrm{CO}_{2}$ reduction. Nat Rev Chem, 2018, 2: $1-7$

72 Lu Q, Rosen J, Zhou Y, et al. A selective and efficient electrocatalyst for carbon dioxide reduction. Nat Commun, 2014, 5: 3242

73 Rasul S, Anjum DH, Jedidi A, et al. A highly selective copperindium bimetallic electrocatalyst for the electrochemical reduction of aqueous $\mathrm{CO}_{2}$ to CO. Angew Chem Int Ed, 2015, 54: 21462150

74 Yin Z, Gao D, Yao S, et al. Highly selective palladium-copper bimetallic electrocatalysts for the electrochemical reduction of $\mathrm{CO}_{2}$ to CO. Nano Energy, 2016, 27: 35-43

75 Zhang $\mathrm{X}$, Li F, Zhang Y, et al. Stannate derived bimetallic nanoparticles for electrocatalytic $\mathrm{CO}_{2}$ reduction. J Mater Chem A, 2018, 6: 7851-7858

76 Kim D, Resasco J, Yu Y, et al. Synergistic geometric and electronic effects for electrochemical reduction of carbon dioxide using gold-copper bimetallic nanoparticles. Nat Commun, 2014, 5: 4948

77 Asadi M, Kim K, Liu C, et al. Nanostructured transition metal dichalcogenide electrocatalysts for $\mathrm{CO}_{2}$ reduction in ionic liquid. Science, 2016, 353: 467-470

78 Hong X, Chan K, Tsai C, et al. How doped $\mathrm{MoS}_{2}$ breaks transition-metal scaling relations for $\mathrm{CO}_{2}$ electrochemical reduction. ACS Catal, 2016, 6: 4428-4437

79 Liu X, Yang H, He J, et al. Highly active, durable ultrathin $\mathrm{MoTe}_{2}$ layers for the electroreduction of $\mathrm{CO}_{2}$ to $\mathrm{CH}_{4}$. Small, 2018, 14: 1704049

80 Asadi M, Kumar B, Behranginia A, et al. Robust carbon dioxide reduction on molybdenum disulphide edges. Nat Commun, 2014, 5: 4470

81 Vasileff A, Zheng Y, Qiao SZ. Carbon solving carbon's problems: Recent progress of nanostructured carbon-based catalysts for the electrochemical reduction of $\mathrm{CO}_{2}$. Adv Energy Mater, 2017, 7: 1700759

82 Blake P, Brimicombe PD, Nair RR, et al. Graphene-based liquid crystal device. Nano Lett, 2009, 8: 1704-1708

83 Eda G, Fanchini G, Chhowalla M. Large-area ultrathin films of reduced graphene oxide as a transparent and flexible electronic material. Nat Nanotech, 2008, 3: 270-274

84 Duan X, Xu J, Wei Z, et al. Metal-free carbon materials for $\mathrm{CO}_{2}$ electrochemical reduction. Adv Mater, 2017, 29: 1701784

85 Zhu Y, Murali S, Stoller MD, et al. Carbon-based supercapacitors produced by activation of graphene. Science, 2011, 332: 15371541

86 Li W, Seredych M, Rodríguez-Castellón E, et al. Metal-free nanoporous carbon as a catalyst for electrochemical reduction of $\mathrm{CO}_{2}$ to $\mathrm{CO}$ and $\mathrm{CH}_{4}$. ChemSusChem, 2016, 9: 606-616

87 Xie J, Zhao X, Wu M, et al. Metal-free fluorine-doped carbon electrocatalyst for $\mathrm{CO}_{2}$ reduction outcompeting hydrogen evolution. Angew Chem Int Ed, 2018, 57: 9640-9644

88 Li W, Bandosz TJ. Role of heteroatoms in S,N-codoped nanoporous carbon materials in $\mathrm{CO}_{2}$ (photo)electrochemical reduc- 
tion. ChemSusChem, 2018, 11: 2987-2999

89 Liu Y, Chen S, Quan X, et al. Efficient electrochemical reduction of carbon dioxide to acetate on nitrogen-doped nanodiamond. J Am Chem Soc, 2015, 137: 11631-11636

90 Zou X, Liu M, Wu J, et al. How nitrogen-doped graphene quantum dots catalyze electroreduction of $\mathrm{CO}_{2}$ to hydrocarbons and oxygenates. ACS Catal, 2017, 7: 6245-6250

91 Yang HP, Lin Q, Zhang HW, et al. Selective electrochemical reduction of $\mathrm{CO}_{2}$ by a binder-free platinum/nitrogen-doped carbon nanofiber/copper foil catalyst with remarkable efficiency and reusability. Electrochem Commun, 2018, 93: 138-142

92 Sun X, Lu L, Zhu Q, et al. MoP nanoparticles supported on indium-doped porous carbon: outstanding catalysts for highly efficient $\mathrm{CO}_{2}$ electroreduction. Angew Chem Int Ed, 2018, 57: 2427-2431

93 Varela AS, Ju W, Strasser P. Molecular nitrogen-carbon catalysts, solid metal organic framework catalysts, and solid metal/nitrogen-doped carbon (MNC) catalysts for the electrochemical $\mathrm{CO}_{2}$ reduction. Adv Energy Mater, 2018, 8: 1703614

94 Baturina OA, Lu Q, Padilla MA, et al. $\mathrm{CO}_{2}$ electroreduction to hydrocarbons on carbon-supported $\mathrm{Cu}$ nanoparticles. ACS Catal, 2014, 4: 3682-3695

95 Varela AS, Ranjbar Sahraie N, Steinberg J, et al. Metal-doped nitrogenated carbon as an efficient catalyst for direct $\mathrm{CO}_{2}$ electroreduction to $\mathrm{CO}$ and hydrocarbons. Angew Chem Int Ed, 2015, 54: 10758-10762

96 Ju W, Bagger A, Hao GP, et al. Understanding activity and selectivity of metal-nitrogen-doped carbon catalysts for electrochemical reduction of $\mathrm{CO}_{2}$. Nat Commun, 2017, 8: 944

97 Pan $\mathrm{F}$, Zhang $\mathrm{H}$, Liu $\mathrm{K}$, et al. Unveiling active sites of $\mathrm{CO}_{2}$ reduction on nitrogen-coordinated and atomically dispersed iron and cobalt catalysts. ACS Catal, 2018, 8: 3116-3122

98 Zhang $\mathrm{C}$, Yang S, Wu J, et al. Electrochemical $\mathrm{CO}_{2}$ reduction with atomic iron-dispersed on nitrogen-doped graphene. Adv Energy Mater, 2018, 8: 1703487

99 Rao H, Schmidt LC, Bonin J, et al. Visible-light-driven methane formation from $\mathrm{CO}_{2}$ with a molecular iron catalyst. Nature, 2014, 548: 74-77

100 Elgrishi N, Chambers MB, Wang X, et al. Molecular polypyridine-based metal complexes as catalysts for the reduction of $\mathrm{CO}_{2}$. Chem Soc Rev, 2017, 46: 761-796

101 Grice KA, Saucedo C. Electrocatalytic reduction of $\mathrm{CO}_{2}$ by group $6 \mathrm{M}(\mathrm{CO})_{6}$ species without "non-innocent" ligands. Inorg Chem, 2016, 55: 6240-6246

102 Bourrez M, Molton F, Chardon-Noblat S, et al. [Mn(bipyridyl) $(\mathrm{CO})_{3} \mathrm{Br}$ ]: An abundant metal carbonyl complex as efficient electrocatalyst for $\mathrm{CO}_{2}$ reduction. Angew Chem Int Ed, 2011, 50: 9903-9906

103 Takeda $\mathrm{H}$, Cometto C, Ishitani O, et al. Electrons, photons, protons and earth-abundant metal complexes for molecular catalysis of $\mathrm{CO}_{2}$ reduction. ACS Catal, 2017, 7: 70-88

104 Azcarate I, Costentin C, Robert M, et al. Through-space charge interaction substituent effects in molecular catalysis leading to the design of the most efficient catalyst of $\mathrm{CO}_{2}$-to- $\mathrm{CO}$ electrochemical conversion. J Am Chem Soc, 2016, 138: 16639-16644

105 Costentin C, Drouet S, Robert M, et al. A local proton source enhances $\mathrm{CO}_{2}$ electroreduction to $\mathrm{CO}$ by a molecular Fe catalyst. Science, 2012, 338: 90-94

106 Costentin C, Passard G, Robert M, et al. Ultraefficient homogeneous catalyst for the $\mathrm{CO}_{2}$-to- $\mathrm{CO}$ electrochemical conversion.
Proc Natl Acad Sci USA, 2014, 111: 14990-14994

107 Costentin C, Robert M, Savéant JM, et al. Efficient and selective molecular catalyst for the $\mathrm{CO}_{2}$-to-CO electrochemical conversion in water. Proc Natl Acad Sci USA, 2015, 112: 6882-6886

108 Bullock RM, Das AK, Appel AM. Surface immobilization of molecular electrocatalysts for energy conversion. Chem Eur J, 2017, 23: 7626-7641

109 Sun C, Prosperini S, Quagliotto P, et al. Electrocatalytic reduction of $\mathrm{CO}_{2}$ by thiophene-substituted rhenium(I) complexes and by their polymerized films. Dalton Trans, 2016, 45: 14678-14688

110 Liu X, Inagaki S, Gong J. Heterogeneous molecular systems for photocatalytic $\mathrm{CO}_{2}$ reduction with water oxidation. Angew Chem Int Ed, 2016, 55: 14924-14950

111 Shen J, Kortlever R, Kas R, et al. Electrocatalytic reduction of carbon dioxide to carbon monoxide and methane at an immobilized cobalt protoporphyrin. Nat Commun, 2015, 6: 8177

112 Choi J, Wagner P, Jalili R, et al. A porphyrin/graphene framework: A highly efficient and robust electrocatalyst for carbon dioxide reduction. Adv Energy Mater, 2018, 8: 1801280

113 Sun C, Rotundo L, Garino C, et al. Electrochemical $\mathrm{CO}_{2}$ reduction at glassy carbon electrodes functionalized by $\mathrm{Mn}^{\mathrm{I}}$ and $\mathrm{Re}^{\mathrm{I}}$ organometallic complexes. ChemPhysChem, 2017, 18: 3219-3229

114 Hu XM, Rønne MH, Pedersen SU, et al. Enhanced catalytic activity of cobalt porphyrin in $\mathrm{CO}_{2}$ electroreduction upon immobilization on carbon materials. Angew Chem, 2017, 129: 65686572

115 Kosal ME, Chou JH, Wilson SR, et al. A functional zeolite analogue assembled from metalloporphyrins. Nat Mater, 2002, 1: 118-121

116 Abrahams BF, Hoskins BF, Michail DM, et al. Assembly of porphyrin building blocks into network structures with large channels. Nature, 1994, 369: 727-729

117 Lee CY, Farha OK, Hong BJ, et al. Light-harvesting metal-organic frameworks (MOFs): Efficient strut-to-strut energy transfer in bodipy and porphyrin-based MOFs. J Am Chem Soc, 2011, 133: 15858-15861

118 Smith PT, Benke BP, Cao Z, et al. Iron porphyrins embedded into a supramolecular porous organic cage for electrochemical $\mathrm{CO}_{2}$ reduction in water. Angew Chem Int Ed, 2018, 57: 9684-9688

119 Fateeva A, Chater PA, Ireland CP, et al. A water-stable porphyrinbased metal-organic framework active for visible-light photocatalysis. Angew Chem Int Ed, 2012, 51: 7440-7444

120 Wan S, Gándara F, Asano A, et al. Covalent organic frameworks with high charge carrier mobility. Chem Mater, 2011, 23: 40944097

121 Lin S, Diercks CS, Zhang YB, et al. Covalent organic frameworks comprising cobalt porphyrins for catalytic $\mathrm{CO}_{2}$ reduction in water. Science, 2015, 349: 1208-1213

122 Behar D, Dhanasekaran T, Neta $\mathrm{P}$, et al. Cobalt porphyrin catalyzed reduction of $\mathrm{CO}_{2}$. Radiation chemical, photochemical, and electrochemical studies. J Phys Chem A, 1998, 102: 2870-2877

123 Pander Iii JE, Fogg A, Bocarsly AB. Utilization of electropolymerized films of cobalt porphyrin for the reduction of carbon dioxide in aqueous media. ChemCatChem, 2016, 8: 3536-3545

124 Lin CY, Zhang D, Zhao Z, et al. Covalent organic framework electrocatalysts for clean energy conversion. Adv Mater, 2018, 30: 1703646

125 Diercks CS, Lin S, Kornienko N, et al. Reticular electronic tuning of porphyrin active sites in covalent organic frameworks for electrocatalytic carbon dioxide reduction. J Am Chem Soc, 2018, 
140: $1116-1122$

126 Shultz AM, Farha OK, Hupp JT, et al. A catalytically active, permanently microporous MOF with metalloporphyrin struts. J Am Chem Soc, 2009, 131: 4204-4205

127 Ahrenholtz SR, Epley CC, Morris AJ. Solvothermal preparation of an electrocatalytic metalloporphyrin MOF thin film and its redox hopping charge-transfer mechanism. J Am Chem Soc, 2014, 136: 2464-2472

128 Morris W, Volosskiy B, Demir S, et al. Synthesis, structure, and metalation of two new highly porous zirconium metal-organic frameworks. Inorg Chem, 2012, 51: 6443-6445

129 Hod I, Sampson MD, Deria P, et al. Fe-porphyrin-based metalorganic framework films as high-surface concentration, heterogeneous catalysts for electrochemical reduction of $\mathrm{CO}_{2}$. ACS Catal, 2015, 5: 6302-6309

130 Dong BX, Qian SL, Bu FY, et al. Electrochemical reduction of $\mathrm{CO}_{2}$ to $\mathrm{CO}$ by a heterogeneous catalyst of Fe-porphyrin-based metal-organic framework. ACS Appl Energy Mater, 2018, 1: 4662-4669

131 Kornienko N, Zhao Y, Kley CS, et al. Metal-organic frameworks for electrocatalytic reduction of carbon dioxide. J Am Chem Soc, 2015, 137: 14129-14135

132 Hod I, Bury W, Karlin DM, et al. Directed growth of electroactive metal-organic framework thin films using electrophoretic deposition. Adv Mater, 2014, 26: 6295-6300

133 Nielsen IMB, Leung K. Cobalt-porphyrin catalyzed electrochemical reduction of carbon dioxide in water. 1. A density functional study of intermediates. J Phys Chem A, 2010, 114: 10166-10173

134 Shen J, Kolb MJ, Göttle AJ, et al. DFT study on the mechanism of the electrochemical reduction of $\mathrm{CO}_{2}$ catalyzed by cobalt porphyrins. J Phys Chem C, 2016, 120: 15714-15721

135 Ye L, Liu J, Gao Y, et al. Highly oriented MOF thin film-based electrocatalytic device for the reduction of $\mathrm{CO}_{2}$ to $\mathrm{CO}$ exhibiting high faradaic efficiency. J Mater Chem A, 2016, 4: 15320-15326

136 Benson EE, Kubiak CP. Structural investigations into the deactivation pathway of the $\mathrm{CO}_{2}$ reduction electrocatalyst $\mathrm{Re}$ (bpy) (CO) ${ }_{3} \mathrm{Cl}$. Chem Commun, 2012, 48: 7374-7376

137 Yao CL, Li JC, Gao W, et al. An integrated design with new metal-functionalized covalent organic frameworks for the effective electroreduction of $\mathrm{CO}_{2}$. Chem Eur J, 2018, 24: 11051-11058

138 Tian Z, Priest C, Chen L. Recent progress in the theoretical investigation of electrocatalytic reduction of $\mathrm{CO}_{2}$. Adv Theor Simul, 2018, 1: 1800004

139 Hernández S, Amin Farkhondehfal M, Sastre F, et al. Syngas production from electrochemical reduction of $\mathrm{CO}_{2}$ : Current status and prospective implementation. Green Chem, 2017, 19: 2326-2346

140 Hori Y, Wakebe H, Tsukamoto T, et al. Electrocatalytic process of $\mathrm{CO}$ selectivity in electrochemical reduction of $\mathrm{CO}_{2}$ at metal electrodes in aqueous media. Electrochim Acta, 1994, 39: 18331839

141 Loiudice A, Lobaccaro P, Kamali EA, et al. Tailoring copper nanocrystals towards $\mathrm{C}_{2}$ products in electrochemical $\mathrm{CO}_{2}$ reduction. Angew Chem Int Ed, 2016, 55: 5789-5792

$142 \mathrm{Gu}$ Z, Shen H, Shang L, et al. Nanostructured copper-based electrocatalysts for $\mathrm{CO}_{2}$ reduction. Small Methods, 2018, 2: 1800121

143 Hinogami R, Yotsuhashi S, Deguchi M, et al. Electrochemical reduction of carbon dioxide using a copper rubeanate metal or- ganic framework. ECS Electrochem Lett, 2012, 1: H17-H19

144 Senthil Kumar R, Senthil Kumar S, Anbu Kulandainathan M. Highly selective electrochemical reduction of carbon dioxide using $\mathrm{Cu}$ based metal organic framework as an electrocatalyst. Electrochem Commun, 2012, 25: 70-73

145 Dai L, Qin Q, Wang P, et al. Ultrastable atomic copper nanosheets for selective electrochemical reduction of carbon dioxide. Sci Adv, 2017, 3: e1701069

146 Albo J, Vallejo D, Beobide G, et al. Copper-based metal-organic porous materials for $\mathrm{CO}_{2}$ electrocatalytic reduction to alcohols. ChemSusChem, 2017, 10: 1100-1109

147 Albo J, Irabien A. $\mathrm{Cu}_{2} \mathrm{O}$-loaded gas diffusion electrodes for the continuous electrochemical reduction of $\mathrm{CO}_{2}$ to methanol. J Catal, 2015, 343: 232-239

148 Perfecto-Irigaray M, Albo J, Beobide G, et al. Synthesis of heterometallic metal-organic frameworks and their performance as electrocatalyst for $\mathrm{CO}_{2}$ reduction. RSC Adv, 2018, 8: 21092-21099

149 Jiang K, Sandberg RB, Akey AJ, et al. Metal ion cycling of Cu foil for selective $\mathrm{C}-\mathrm{C}$ coupling in electrochemical $\mathrm{CO}_{2}$ reduction. Nat Catal, 2018, 1: 111-119

150 Zhou Y, Che F, Liu M, et al. Dopant-induced electron localization drives $\mathrm{CO}_{2}$ reduction to $\mathrm{C}_{2}$ hydrocarbons. Nat Chem, 2018, 10: 974-980

151 Kang X, Zhu Q, Sun X, et al. Highly efficient electrochemical reduction of $\mathrm{CO}_{2}$ to $\mathrm{CH}_{4}$ in an ionic liquid using a metal-organic framework cathode. Chem Sci, 2016, 7: 266-273

152 Roy N, Shibano Y, Terashima C, et al. Ionic-liquid-assisted selective and controlled electrochemical $\mathrm{CO}_{2}$ reduction at $\mathrm{Cu}$ modified boron-doped diamond electrode. ChemElectroChem, 2016, 3: 1044-1047

153 Park KS, Ni Z, Côté AP, et al. Exceptional chemical and thermal stability of zeolitic imidazolate frameworks. Proc Natl Acad Sci USA, 2006, 103: 10186-10191

154 Venna SR, Jasinski JB, Carreon MA. Structural evolution of zeolitic imidazolate framework-8. J Am Chem Soc, 2010, 132: 18030-18033

155 Huang XC, Lin YY, Zhang JP, et al. Ligand-directed strategy for zeolite-type metal-organic frameworks: Zinc(II) imidazolates with unusual zeolitic topologies. Angew Chem Int Ed, 2006, 45: 1557-1559

156 Cravillon J, Munzer S, Lohmeier SJ, et al. Rapid room-temperature synthesis and characterization of nanocrystals of a prototypical zeolitic imidazolate framework. Chem Mater, 2009, 21: 1410-1412

157 Pan Y, Liu Y, Zeng G, et al. Rapid synthesis of zeolitic imidazolate framework-8 (ZIF-8) nanocrystals in an aqueous system. Chem Commun, 2011, 47: 2071-2073

158 Lin JB, Lin RB, Cheng XN, et al. Solvent/additive-free synthesis of porous/zeolitic metal azolate frameworks from metal oxide/hydroxide. Chem Commun, 2011, 47: 9185-9187

159 Wang Y, Hou P, Wang Z, et al. Zinc imidazolate metal-organic frameworks (ZIF-8) for electrochemical reduction of $\mathrm{CO}_{2}$ to $\mathrm{CO}$. ChemPhysChem, 2017, 18: 3142-3147

160 Rosen BA, Hod I. Tunable molecular-scale materials for catalyzing the low-overpotential electrochemical conversion of $\mathrm{CO}_{2}$. Adv Mater, 2018, 30: 1706238

161 Liédana N, Galve A, Rubio C, et al. CAF@ZIF-8: One-step encapsulation of caffeine in MOF. ACS Appl Mater Interfaces, 2012, 4: 5016-5021

162 Rungtaweevoranit B, Baek J, Araujo JR, et al. Copper nanocrystals 
encapsulated in $\mathrm{Zr}$-based metal-organic frameworks for highly selective $\mathrm{CO}_{2}$ hydrogenation to methanol. Nano Lett, 2016, 16: 7645-7649

163 Zhang SY, Yang YY, Zheng YQ, et al. Ag-doped $\mathrm{Co}_{3} \mathrm{O}_{4}$ catalyst derived from heterometallic $\mathrm{MOF}$ for syngas production by electrocatalytic reduction of $\mathrm{CO}_{2}$ in water. J Solid State Chem, 2018, 263: 44-51

164 Kung CW, Audu CO, Peters AW, et al. Copper nanoparticles installed in metal-organic framework thin films are electrocatalytically competent for $\mathrm{CO}_{2}$ reduction. ACS Energy Lett, 2017, 2: 2394-2401

165 Wang TC, Vermeulen NA, Kim IS, et al. Scalable synthesis and post-modification of a mesoporous metal-organic framework called NU-1000. Nat Protoc, 2016, 11: 149-162

166 Kopljar D, Wagner N, Klemm E. Transferring electrochemical $\mathrm{CO}_{2}$ reduction from semi-batch into continuous operation mode using gas diffusion electrodes. Chem Eng Technol, 2016, 39: 2042-2050

167 Marepally BC, Ampelli C, Genovese C, et al. Enhanced formation of $>\mathrm{Cl}$ products in electroreduction of $\mathrm{CO}_{2}$ by adding a $\mathrm{CO}_{2}$ adsorption component to a gas-diffusion layer-type catalytic electrode. ChemSusChem, 2017, 10: 4442-4446

168 Weng LC, Bell AT, Weber AZ. Modeling gas-diffusion electrodes for $\mathrm{CO}_{2}$ reduction. Phys Chem Chem Phys, 2018, 20: 1697316984

169 Liu M, Pang Y, Zhang B, et al. Enhanced electrocatalytic $\mathrm{CO}_{2}$ reduction via field-induced reagent concentration. Nature, 2016, 537: $382-386$

170 Rodríguez-Albelo LM, López-Maya E, Hamad S, et al. Selective sulfur dioxide adsorption on crystal defect sites on an isoreticular metal organic framework series. Nat Commun, 2017, 8: 14457

171 Liu G, Chernikova V, Liu Y, et al. Mixed matrix formulations with MOF molecular sieving for key energy-intensive separations. Nat Mater, 2018, 17: 283-289

172 Han X, Godfrey HGW, Briggs L, et al. Reversible adsorption of nitrogen dioxide within a robust porous metal-organic framework. Nat Mater, 2018, 17: 691-696

173 Carrington EJ, McAnally CA, Fletcher AJ, et al. Solvent-switchable continuous-breathing behaviour in a diamondoid metal-organic framework and its influence on $\mathrm{CO}_{2}$ versus $\mathrm{CH}_{4}$ selectivity. Nat Chem, 2017, 9: 882-889

174 Shekhah O, Belmabkhout Y, Chen Z, et al. Made-to-order metalorganic frameworks for trace carbon dioxide removal and air capture. Nat Commun, 2014, 5: 4228

175 Liang L, Liu C, Jiang F, et al. Carbon dioxide capture and conversion by an acid-base resistant metal-organic framework. Nat Commun, 2017, 8: 1233

176 Ghalei B, Sakurai K, Kinoshita Y, et al. Enhanced selectivity in mixed matrix membranes for $\mathrm{CO}_{2}$ capture through efficient dispersion of amine-functionalized MOF nanoparticles. Nat Energy, 2017, 2: 17086

177 Yang S, Sun J, Ramirez-Cuesta AJ, et al. Selectivity and direct visualization of carbon dioxide and sulfur dioxide in a decorated porous host. Nat Chem, 2012, 4: 887-894

178 Qiu YL, Zhong HX, Zhang TT, et al. Selective electrochemical reduction of carbon dioxide using $\mathrm{Cu}$ based metal organic framework for $\mathrm{CO}_{2}$ capture. ACS Appl Mater Interfaces, 2018, 10: 2480-2489

179 Blinder SM, Nordman CE. Collision theory of chemical reactions. J Chem Educ, 1974, 51: 790-791
180 Liu H, Chu J, Yin Z, et al. Covalent organic frameworks linked by amine bonding for concerted electrochemical reduction of $\mathrm{CO}_{2}$. Chem, 2018, 4: 1696-1709

181 Li P, Zeng HC. Advanced oxygen evolution catalysis by bimetallic $\mathrm{Ni}-\mathrm{Fe}$ phosphide nanoparticles encapsulated in nitrogen, phosphorus, and sulphur tri-doped porous carbon. Chem Commun, 2017, 53: 6025-6028

182 Zhang H, Liu X, Wu Y, et al. MOF-derived nanohybrids for electrocatalysis and energy storage: Current status and perspectives. Chem Commun, 2018, 54: 5268-5288

183 Yilmaz G, Yam KM, Zhang C, et al. In situ transformation of MOFs into layered double hydroxide embedded metal sulfides for improved electrocatalytic and supercapacitive performance. Adv Mater, 2017, 29: 1606814

184 Jiang Y, Liu H, Tan X, et al. Monoclinic ZIF-8 nanosheet-derived 2D carbon nanosheets as sulfur immobilizer for high-performance lithium sulfur batteries. ACS Appl Mater Interfaces, 2017, 9: 25239-25249

185 Li W, Hu S, Luo X, et al. Confined amorphous red phosphorus in MOF-derived $\mathrm{N}$-doped microporous carbon as a superior anode for sodium-ion battery. Adv Mater, 2017, 29: 1605820

186 Nam DH, Bushuyev OS, Li J, et al. Metal-organic frameworks mediate $\mathrm{Cu}$ coordination for selective $\mathrm{CO}_{2}$ electroreduction. J Am Chem Soc, 2018, 140: 11378-11386

187 Chung DY, Lee KJ, Yu SH, et al. Alveoli-inspired facile transport structure of $\mathrm{N}$-doped porous carbon for electrochemical energy applications. Adv Energy Mater, 2015, 5: 1401309

188 Zhong S, Zhan C, Cao D. Zeolitic imidazolate framework-derived nitrogen-doped porous carbons as high performance supercapacitor electrode materials. Carbon, 2015, 85: 51-59

189 Wang R, Sun X, Ould-Chikh S, et al. Metal-organic-frameworkmediated nitrogen-doped carbon for $\mathrm{CO}_{2}$ electrochemical reduction. ACS Appl Mater Interfaces, 2018, 10: 14751-14758

190 Zhang YZ, Wang Y, Xie YL, et al. Porous hollow $\mathrm{Co}_{3} \mathrm{O}_{4}$ with rhombic dodecahedral structures for high-performance supercapacitors. Nanoscale, 2014, 6: 14354-14359

191 Huang G, Zhang F, Du X, et al. Metal organic frameworks route to in situ insertion of multiwalled carbon nanotubes in $\mathrm{Co}_{3} \mathrm{O}_{4}$ polyhedra as anode materials for lithium-ion batteries. ACS Nano, 2015, 9: 1592-1599

192 Wang Y, Chen B, Chang Z, et al. Enhancing performance of sandwich-like cobalt sulfide and carbon for quasi-solid-state hybrid electrochemical capacitors. J Mater Chem A, 2017, 5: 89818988

193 Wei J, Hu Y, Liang Y, et al. Nitrogen-doped nanoporous carbon/ graphene nano-sandwiches: Synthesis and application for efficient oxygen reduction. Adv Funct Mater, 2015, 25: 5768-5777

194 Qu Q, Gao T, Zheng H, et al. Graphene oxides-guided growth of ultrafine $\mathrm{Co}_{3} \mathrm{O}_{4}$ nanocrystallites from MOFs as high-performance anode of Li-ion batteries. Carbon, 2015, 92: 119-125

195 Wang Y, Chen B, Zhang Y, et al. ZIF-8@MWCNT-derived carbon composite as electrode of high performance for supercapacitor. Electrochim Acta, 2016, 213: 260-269

196 Guo Y, Yang H, Zhou X, et al. Electrocatalytic reduction of $\mathrm{CO}_{2}$ to $\mathrm{CO}$ with $100 \%$ faradaic efficiency by using pyrolyzed zeolitic imidazolate frameworks supported on carbon nanotube networks. J Mater Chem A, 2017, 5: 24867-24873

197 Zhang P, Zhu H, Dai S. Porous carbon supports: Recent advances with various morphologies and compositions. ChemCatChem, 2015, 7: 2788-2805 

nanofibers incorporated with $\mathrm{NiO}$ nanoparticles as free-standing film electrodes for high-performance supercapacitors and $\mathrm{CO}_{2}$ capture. Small, 2018, 14: 1704203

199 Jhong HRM, Tornow CE, Smid B, et al. A nitrogen-doped carbon catalyst for electrochemical $\mathrm{CO}_{2}$ conversion to $\mathrm{CO}$ with high selectivity and current density. ChemSusChem, 2017, 10: 10941099

200 Shi JJ, Hu XM, Madsen MR, et al. Facile synthesis of iron- and nitrogen-doped porous carbon for selective $\mathrm{CO}_{2}$ electroreduction. ACS Appl Nano Mater, 2018, 1: 3608-3615

201 Zhao K, Liu Y, Quan X, et al. $\mathrm{CO}_{2}$ electroreduction at low overpotential on oxide-derived $\mathrm{Cu}$ /carbons fabricated from metal organic framework. ACS Appl Mater Interfaces, 2017, 9: 53025311

202 Zhuang TT, Liang ZQ, Seifitokaldani A, et al. Steering post-C-C coupling selectivity enables high efficiency electroreduction of carbon dioxide to multi-carbon alcohols. Nat Catal, 2018, 1: 421428

203 Huang R, Peng Y, Wang C, et al. A rhenium-functionalized metal-organic framework as a single-site catalyst for photochemical reduction of carbon dioxide. Eur J Inorg Chem, 2016, 2016: 4358-4362

204 Thomas JM, Raja R, Lewis DW. Single-site heterogeneous catalysts. Angew Chem Int Ed, 2005, 44: 6456-6482

205 Wang X, Chen Z, Zhao X, et al. Regulation of coordination number over single Co sites: Triggering the efficient electroreduction of $\mathrm{CO}_{2}$. Angew Chem Int Ed, 2018, 57: 1944-1948

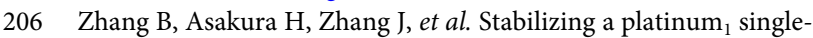
atom catalyst on supported phosphomolybdic acid without compromising hydrogenation activity. Angew Chem Int Ed, 2016, 55: 8319-8323

207 Yin P, Yao T, Wu Y, et al. Single cobalt atoms with precise Ncoordination as superior oxygen reduction reaction catalysts. Angew Chem Int Ed, 2016, 55: 10800-10805

208 Rogge SMJ, Bavykina A, Hajek J, et al. Metal-organic and covalent organic frameworks as single-site catalysts. Chem Soc Rev, 2017, 46: $3134-3184$

209 Canivet J, Aguado S, Schuurman Y, et al. MOF-supported selective ethylene dimerization single-site catalysts through one-pot postsynthetic modification. J Am Chem Soc, 2013, 135: 41954198

210 Li Z, Schweitzer NM, League AB, et al. Sintering-resistant singlesite nickel catalyst supported by metal-organic framework. J Am Chem Soc, 2016, 138: 1977-1982

211 Zhao C, Dai X, Yao T, et al. Ionic exchange of metal-organic frameworks to access single nickel sites for efficient electroreduction of $\mathrm{CO}_{2}$. J Am Chem Soc, 2017, 139: 8078-8081

212 Yang HB, Hung SF, Liu S, et al. Atomically dispersed $\mathrm{Ni}(\mathrm{I})$ as the active site for electrochemical $\mathrm{CO}_{2}$ reduction. Nat Energy, 2018, 3: $140-147$

213 Qiu HJ, Ito Y, Cong W, et al. Nanoporous graphene with singleatom nickel dopants: An efficient and stable catalyst for electrochemical hydrogen production. Angew Chem, 2015, 127: 1423714241

214 Huan TN, Ranjbar N, Rousse G, et al. Electrochemical reduction of $\mathrm{CO}_{2}$ catalyzed by Fe-N-C materials: A structure-selectivity study. ACS Catal, 2017, 7: 1520-1525

215 Zitolo A, Goellner V, Armel V, et al. Identification of catalytic sites for oxygen reduction in iron- and nitrogen-doped graphene materials. Nat Mater, 2015, 14: 937-942

216 Yan $\mathrm{C}$, Ye Y, Lin L, et al. Improving $\mathrm{CO}_{2}$ electroreduction over ZIF-derived carbon doped with $\mathrm{Fe}-\mathrm{N}$ sites by an additional ammonia treatment. Catal Today, 2019, 330: 252-258

217 Yang J, Zhang F, Lu H, et al. Hollow Zn/Co ZIF particles derived from core-shell ZIF-67@ZIF-8 as selective catalyst for the semihydrogenation of acetylene. Angew Chem Int Ed, 2015, 54: 10889-10893

218 Wright AM, Rieth AJ, Yang S, et al. Precise control of pore hydrophilicity enabled by post-synthetic cation exchange in metalorganic frameworks. Chem Sci, 2018, 9: 3856-3859

219 Song X, Zhang H, Yang Y, et al. Bifunctional nitrogen and cobalt codoped hollow carbon for electrochemical syngas production. Adv Sci, 2018, 5: 1800177

220 Schoedel A, Ji Z, Yaghi OM. The role of metal-organic frameworks in a carbon-neutral energy cycle. Nat Energy, 2016, 1: 16034

221 Trickett CA, Helal A, Al-Maythalony BA, et al. The chemistry of metal-organic frameworks for $\mathrm{CO}_{2}$ capture, regeneration and conversion. Nat Rev Mater, 2017, 2: 17045

222 Diercks CS, Liu Y, Cordova KE, et al. The role of reticular chemistry in the design of $\mathrm{CO}_{2}$ reduction catalysts. Nat Mater, 2018, 17: 301-307

223 He CT, Jiang L, Ye ZM, et al. Exceptional hydrophobicity of a large-pore metal-organic zeolite. J Am Chem Soc, 2015, 137: 7217-7223

224 Busche MR, Drossel T, Leichtweiss T, et al. Dynamic formation of a solid-liquid electrolyte interphase and its consequences for hybrid-battery concepts. Nat Chem, 2018, 8: 426-434

225 Manthiram A, Yu X, Wang S. Lithium battery chemistries enabled by solid-state electrolytes. Nat Rev Mater, 2017, 2: 16103

226 Wu Y, Jiang J, Weng Z, et al. Electroreduction of $\mathrm{CO}_{2}$ catalyzed by a heterogenized $\mathrm{Zn}$-porphyrin complex with a redox-innocent metal center. ACS Cent Sci, 2017, 3: 847-852

227 Li Y, Chan SH, Sun Q. Heterogeneous catalytic conversion of $\mathrm{CO}_{2}$ : A comprehensive theoretical review. Nanoscale, 2015, 7: 8663-8683

228 Rosen BA, Salehi-Khojin A, Thorson MR, et al. Ionic liquidmediated selective conversion of $\mathrm{CO}_{2}$ to $\mathrm{CO}$ at low overpotentials. Science, 2011, 334: 643-644

229 Ma L, Fan S, Zhen D, et al. Electrochemical reduction of $\mathrm{CO}_{2}$ in proton exchange membrane reactor: The function of buffer layer. Ind Eng Chem Res, 2017, 56: 10242-10250

Acknowledgements This work was financially supported by the National Natural Science Foundation of China (21671096 and 11775105), and Shenzhen Peacock Plan (KQTD2016022620054656).

Author contributions Wang $\mathrm{Y}$ and Li Y conceived and wrote the paper under the supervision of Lu Z. Wang Z, Allan P and Zhang F helped in the revision of this review. All authors contributed to the general discussion.

Conflict of interest The authors declare that they have no conflict of interest. 


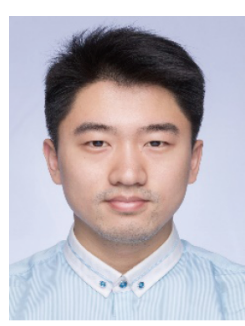

Yanfang Wang received his BE degree from Central South University in 2014 and MS degree from Fudan University in 2017. Now, he is a PhD student at Southern University of Science and Technology (SUSTech), China, jointly with the University of Birmingham, UK. His research interests mainly focus on supercapacitors and lithium-ion batteries.

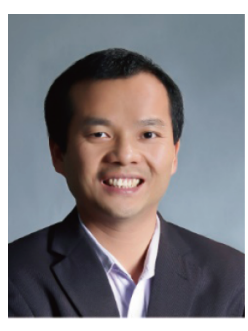

Zhouguang Lu is currently a professor in the Department of Materials Science and Engineering, SUSTech, China. He received his $\mathrm{PhD}$ degree from the City University of Hong Kong in 2009. $\mathrm{He}$ is the recipient of Fulbright Fellowship of USA Government in 2008-2009 and the Overseas High-Caliber Personnel (Level B) of Shenzhen Government in 2013. His research mainly covers the design and synthesis of nanostructures and their applications in energy storage and conversion with focus on lithium/sodium-ion and -air batteries. He has authored more than 160 peer-review journal papers with total citations more than 5600 and $\mathrm{H}$-index of 46 .

\section{框架化学在电化学还原二氧化碳中的应用}

王彦方 ${ }^{1,2,4 \dagger}$, 李月香 ${ }^{3 \dagger}$, 王振宇 ${ }^{1}$, Phoebe Allan ${ }^{4^{*}}$, 张福才 ${ }^{2^{*}}$, 卢周广

摘要 利用电化学方法将大气中的二氧化碳还原成具有工业价值 的原材料被认为是缓解温室效应、实现碳中和的重要手段. 然而, 电化学还原二氧化碳的过程涉及多种复杂的反应, 寻找和开发高 效的电化学催化剂被认为是推动该领域发展的工作重点. 近年来, 金属有机框架结构和共价有机框架结构因其超高的比表面积、可 调控的孔道结构等特征被广泛应用在各个领域. 同时, 随之发展的 框架化学为从原子/分子级设计具有特定功能的有机框架结构提供 了理论基础. 本文综述了近年来有机框架结构及其衍生材料在电 化学二氧化碳还原方向的应用, 并展望了框架化学在该领域中的 挑战、机遇以及发展方向. 